\title{
Clique-width and well-quasi-ordering of triangle-free graph classes
}

\author{
Konrad K. Dabrowski ${ }^{\mathrm{a}, *}$, Vadim V. Lozin ${ }^{\mathrm{b}}$, Daniël Paulusma ${ }^{\mathrm{a}}$ \\ a Department of Computer Science, Durham University, Lower Mountjoy, South Road, Durham DH1 3LE, United Kingdom \\ ${ }^{\mathrm{b}}$ Mathematics Institute, University of Warwick, Coventry CV4 7AL, United Kingdom
}

\section{A R T I C L E I N F O}

\section{Article history:}

Received 24 May 2018

Received in revised form 29 December 2018

Accepted 9 September 2019

Available online $\mathrm{xxxx}$

\section{Keywords:}

Clique-width

Forbidden induced subgraph

Hereditary graph class

Well-quasi-ordering

\begin{abstract}
A B S T R A C T
We obtain a complete classification of graphs $H$ for which the class of (triangle, $H$ )-free graphs is well-quasi-ordered by the induced subgraph relation and an almost complete classification of graphs $H$ for which the class of (triangle, $H$ )-free graphs has bounded clique-width. In particular, we show that for these graph classes, well-quasi-orderability implies boundedness of clique-width. To obtain our results, we further refine a known method based on canonical decomposition. This leads to a new decomposition technique that is applicable to both notions, well-quasi-orderability and clique-width.
\end{abstract}

(C) 2019 The Authors. Published by Elsevier Inc. This is an open access article under the CC BY license (http://creativecommons.org/licenses/by/4.0/).

\section{Introduction}

Clique-width. Every NP-hard graph problem becomes polynomial-time solvable after placing appropriate restrictions on the input. A general method in the design of graph algorithms for special graph classes is to decompose the vertex set of the graph into large sets of "similarly behaving"' vertices and to exploit this decomposition algorithmically. An optimal vertex set decomposition gives us the "width" of the graph. Clique-width is one of the most studied width parameters (see, for example, the surveys $[26,29])$. The vertex set decomposition of clique-width is defined via a graph construction based on vertex labellings. Starting from the empty graph, a graph $G$ is built up vertex-by-vertex using four specific graph operations. These operations ensure that vertices labelled alike will keep the same label and thus "behave" identically. The clique-width of $G$ is the minimum number of different labels needed to construct $G$ in this way (see Section 2 for a precise definition). A graph class $\mathcal{G}$ has bounded clique-width if there exists a constant $c$ such that every graph in $\mathcal{G}$ has clique-width at most $c$. The algorithmic importance of having bounded clique-width follows from the existence of several meta-theorems [10,21,31, 41] which, when combined with an approximation result [39], ensure that many well-known NP-hard graph problems, such as Graph Colouring and HAmilton CyCLE, become polynomial-time solvable for every graph class of bounded clique-width. Hence, there is a need to verify boundedness of clique-width of special graph classes, in particular when undertaking a systematic classification into the computational complexity of graph problems under input restrictions; see, for example, [25] for the importance of clique-width for the GRAPH COLOURING problem.

\footnotetext{
This research was supported by EPSRC (EP/K025090/1 and EP/L020408/1) and the Leverhulme Trust RPG-2016-258. An extended abstract of this paper appeared in the proceedings of WG 2017 [14].

* Corresponding author.

E-mail addresses: konrad.dabrowski@durham.ac.uk (K.K. Dabrowski), v.lozin@warwick.ac.uk (V.V. Lozin), daniel.paulusma@durham.ac.uk (D. Paulusma).
} 
Well-quasi-orderings. A graph class $\mathcal{G}$ is well-quasi-ordered by a containment relation if for any infinite sequence $G_{0}, G_{1}, \ldots$ of graphs in $\mathcal{G}$, there is a pair $i, j$ with $i<j$ such that $G_{i}$ is contained in $G_{j}$. Just as is the case for having bounded clique-width, being well-quasi-ordered is a highly desirable property, which has been frequently discovered in the areas of discrete mathematics and theoretical computer science [1,23,35]. To illustrate its importance, let us mention the seminal project of Robertson and Seymour on graph minors, which culminated in 2004 in the proof [43] of Wagner's conjecture. Wagner's conjecture states that the set of all finite graphs is well-quasi-ordered by the minor relation (a graph $H$ is a minor of a graph $G$ if $H$ can be obtained from $G$ via a sequence of vertex deletions, edge deletions and edge contractions). As an algorithmic consequence, given a minor-closed graph class, it is possible to test in cubic time whether a given graph belongs to this class [42] (see [28] for a quadratic algorithm).

To give some more examples, a result of Ding [20] implies that every class of graphs with bounded vertex cover number is well-quasi-ordered by the induced subgraph relation, whereas Mader [38] showed that every class of graphs with bounded feedback vertex number is well-quasi-ordered by the topological minor relation. Fellows, Hermelin and Rosamund [22] simplified the proofs of these results and also showed that every class of graphs of bounded circumference is well-quasi-ordered by the induced minor relation. Furthermore, as "interesting applications" of these three results, they gave linear-time algorithms for recognizing graphs from any topological-minor-closed graph class of bounded feedback vertex number, any induced-minor-closed graph class of bounded circumference, and any induced-subgraph-closed graph class of bounded vertex cover number.

Hereditary graph classes. Graph classes closed under taking induced subgraphs are said to be hereditary. Courcelle [9] proved that the class of graphs obtained from graphs of clique-width 3 via one or more edge contractions has unbounded clique-width. This means that the clique-width of a graph can be much smaller than the clique-width of its minors. On the other hand, the clique-width of a graph is at least the clique-width of any of its induced subgraphs (see, for example, [11]). Hence, it is natural to focus on determining boundedness of clique-width for hereditary graph classes.

Research goals. Nothing in the definitions of clique-width and well-quasi-orderability suggests that there is anything in common between the two notions. However, as we will discuss, recent results for hereditary graph classes suggested an intriguing connection between them. This connection is not yet well understood, and as such, it remains to be further explored. Our underlying research goals are:

- to increase understanding of the relation between well-quasi-orderability by the induced subgraph relation and boundedness of clique-width for hereditary graph classes; and

- to obtain new results for both notions applied to hereditary graph classes.

In a previous paper [15] we showed that certain graph operations and graph constructions work equally well for bounded clique-width and well-quasi-orderability. In this paper we will explore common graph techniques further. Before discussing our results in detail, we first discuss a conjecture that motivated our research.

Conjecture. We first note that the hereditary class of graphs of degree at most 2 is not well-quasi-ordered by the induced subgraph relation, as it contains the class of cycles, which form an infinite antichain. As every graph of degree at most 2 has clique-width at most 4 , having bounded clique-width does not imply well-quasi-orderability by the induced subgraph relation. In 2010, Daligault, Rao and Thomassé [18] asked about the reverse implication: does every hereditary graph class that is well-quasi-ordered by the induced subgraph relation have bounded clique-width? At WG 2015, Lozin, Razgon and Zamaraev [37] gave a negative answer to this question. It is readily seen that a class of graphs is hereditary if and only if it can be characterised by a unique set $\mathcal{F}$ of minimal forbidden induced subgraphs. As the set $\mathcal{F}$ of minimal forbidden induced subgraphs in the counter-example of [37] is infinite, the question of Daligault, Rao and Thomassé [18] remains open for finitely defined hereditary graph classes, that is, hereditary graph classes for which $\mathcal{F}$ is finite.

Conjecture 1 ([37]). If a finitely defined hereditary class of graphs $\mathcal{G}$ is well-quasi-ordered by the induced subgraph relation, then $\mathcal{G}$ has bounded clique-width.

If Conjecture 1 were true, then for finitely defined hereditary graph classes, the aforementioned algorithmic consequences of having bounded clique-width would also hold for the property of being well-quasi-ordered by the induced subgraph relation. In fact, being well-quasi-ordered could be the underlying property enabling these consequences.

A hereditary graph class defined by a single forbidden induced subgraph $H$ is well-quasi-ordered by the induced subgraph relation if and only if it has bounded clique-width if and only if $H$ is an induced subgraph of $P_{4}$ (see, for instance, [17, 19,33]). ${ }^{1}$ Hence Conjecture 1 holds when $\mathcal{F}$ has size 1 . We consider the case when $\mathcal{F}$ has size 2 , say $\mathcal{F}=\left\{H_{1}, H_{2}\right\}$. Such graph classes are said to be bigenic or $\left(H_{1}, H_{2}\right)$-free graph classes. In this case Conjecture 1 is also known to be true except for two stubborn open cases, namely $\left(H_{1}, H_{2}\right)=\left(K_{3}, P_{2}+P_{4}\right)$ and $\left(H_{1}, H_{2}\right)=\left(\overline{P_{1}+P_{4}}, P_{2}+P_{3}\right)$; see [15]. In both of

1 We refer to $[5,20,30]$ for classifications of well-quasi-orderability by the induced-minor, subgraph and contraction relations, respectively, for classes of $H$-free graphs. 


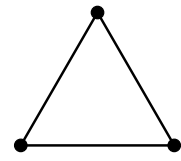

$K_{3}$

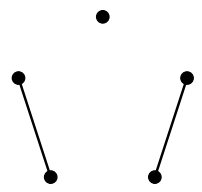

$P_{1}+2 P_{2}$

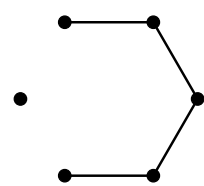

$P_{1}+P_{5}$

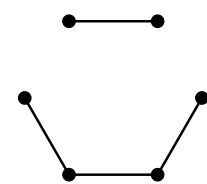

$P_{2}+P_{4}$

Fig. 1. The forbidden induced subgraphs considered in our results.

these cases, it is neither known whether the graph class under consideration has bounded clique-width nor whether it is well-quasi-ordered by the induced subgraph relation.

Results and methodology. In Section 5 , we prove that Conjecture 1 holds for the class of $\left(K_{3}, P_{2}+P_{4}\right)$-free graphs by showing that this class has bounded clique-width and is well-quasi-ordered by the induced subgraph relation. Instead of using ad hoc arguments, we emphasize that our underlying research goal is to develop methodology that could also be applied more widely to other classes and which increases our understanding of the structure of special graph classes. In this paper we focus on $K_{3}$-free graphs and we prove the two results on $\left(K_{3}, P_{2}+P_{4}\right)$-free graphs by using a general method explained in Section 4.

Our method is based on extending (a labelled version of) well-quasi-orderability or boundedness of clique-width of the bipartite graphs in a hereditary graph class $X$ to a special subclass of 3-partite graphs in $X$. The crucial property of these 3 -partite graphs is that no three vertices from the three different partition classes form a clique or independent set. We say that such 3-partite graphs are curious. The method finds its origin in a paper of Fouquet, Giakoumakis and Vanherpe [24], who characterized totally decomposable bipartite graphs, that is, bipartite graphs that can recursively be canonically decomposed into graphs isomorphic to an isolated vertex. Dabrowski, Dross and Paulusma [12] generalized this decomposition from bipartite graphs to $k$-partite graphs for any $k \geq 2$ and partially characterized totally 3-decomposable graphs. This sufficed to prove that $\left(K_{3}, P_{1}+P_{5}\right)$-free graphs have bounded clique-width [12], but it is not strong enough for our purposes. Our new decomposition method allows us to also deal with curious graphs that are not totally 3-decomposable.

In Section 5 we show how to generalize results for curious $\left(K_{3}, P_{2}+P_{4}\right)$-free graphs to the whole class of $\left(K_{3}, P_{2}+P_{4}\right)$-free graphs. Due to the generality of our method, it immediately follows that the class of $\left(K_{3}, P_{1}+P_{5}\right)$-free graphs is also well-quasi-ordered, which was not previously known (see [3,33]). Since $\left(K_{3}, P_{1}+2 P_{2}\right)$-free graphs are $\left(K_{3}, P_{1}+P_{5}\right)$-free (and $\left(K_{3}, P_{2}+P_{4}\right)$-free), our results immediately imply that the class of $\left(K_{3}, P_{1}+2 P_{2}\right)$-free graphs is also well-quasi-ordered by the induced subgraph relation, which was also previously unknown (see [3,33]). As mentioned, it was already known [12] that the class of $\left(K_{3}, P_{1}+P_{5}\right)$-free graphs has bounded clique-width (and so the same was known for the class of $\left(K_{3}, P_{1}+2 P_{2}\right)$-free graphs); as we will see, this also follows directly from our method. See Fig. 1 for pictures of the forbidden induced subgraphs mentioned in this paragraph.

Dichotomy for $K_{3}$-free graphs. Previously, well-quasi-orderability was known for $\left(K_{3}, P_{6}\right)$-free graphs [3], $\left(P_{2}+P_{4}\right)$-free bipartite graphs [32] and $\left(P_{1}+P_{5}\right)$-free bipartite graphs [32]. It has also been shown that $H$-free bipartite graphs are not well-quasi-ordered if $H$ contains an induced $3 P_{1}+P_{2}$ [33], $3 P_{2}$ [20] or $2 P_{3}$ [32]. Moreover, for every $s \geq 1$, the class of $\left(K_{3}, s P_{1}\right)$-free graphs is finite due to Ramsey's Theorem [40]. The above results lead to the following known dichotomy for $H$-free bipartite graphs.

Theorem 1. Let $H$ be a graph. The class of $H$-free bipartite graphs is well-quasi-ordered by the induced subgraph relation if and only if $H=s P_{1}$ for some $s \geq 1$ or $H$ is an induced subgraph of $P_{1}+P_{5}, P_{2}+P_{4}$ or $P_{6}$.

Now combining the aforementioned known results for $\left(K_{3}, H\right)$-free graphs and $H$-free bipartite graphs with our new results yields the following new dichotomy for $\mathrm{H}$-free triangle-free graphs, which is exactly the same as the one in Theorem 1.

Theorem 2. Let $H$ be a graph. The class of $\left(K_{3}, H\right)$-free graphs is well-quasi-ordered by the induced subgraph relation if and only if $H=s P_{1}$ for some $s \geq 1$ or $H$ is an induced subgraph of $P_{1}+P_{5}, P_{2}+P_{4}$, or $P_{6}$.

Besides our method based on curious graphs, we also expect that Theorem 2 will itself be a useful ingredient for showing results on well-quasi-orderability for other graph classes, just as Theorem 1 has already proven to be useful for this (see e.g. [32]).

For clique-width the following dichotomy is known for $\mathrm{H}$-free bipartite graphs; see Section 2 for the definition of the graph $S_{h, i, j}$.

Theorem 3 ([16]). Let $H$ be a graph. The class of $H$-free bipartite graphs has bounded clique-width if and only if $H=s P_{1}$ for some $s \geq 1$ or $H$ is an induced subgraph of $K_{1,3}+3 P_{1}, K_{1,3}+P_{2}, P_{1}+S_{1,1,3}$ or $S_{1,2,3}$. 
It would be interesting to determine whether $\left(K_{3}, H\right)$-free graphs allow the same dichotomy with respect to the boundedness of their clique-width. The evidence so far is affirmative, but in order to answer this question two remaining cases need to be solved, namely $\left(H_{1}, H_{2}\right)=\left(K_{3}, P_{1}+S_{1,1,3}\right)$ and $\left(H_{1}, H_{2}\right)=\left(K_{3}, S_{1,2,3}\right)$. Both cases turn out to be highly nontrivial; in particular, the class of $\left(K_{3}, P_{1}+S_{1,1,3}\right)$-free graphs contains the class of $\left(K_{3}, P_{1}+P_{5}\right)$-free graphs, and the class of $\left(K_{3}, S_{1,2,3}\right)$-free graphs contains both the classes of $\left(K_{3}, P_{1}+P_{5}\right)$-free and $\left(K_{3}, P_{2}+P_{4}\right)$-free graphs.

In Section 6 we give state-of-the-art summaries for well-quasi-orderability and boundedness of clique-width of bigenic graph classes (which include our new results), together with an overview of the missing cases for both problems (which include the missing cases mentioned in this section). In particular, these summaries show that our results for $\left(K_{3}, P_{2}+P_{4}\right)$-free graphs are tight in the sense that $\left(H, P_{2}+P_{4}\right)$-free graphs have unbounded clique-width and are not well-quasi-ordered by the induced subgraph relation for any induced supergraph $H$ of $K_{3}$, unless $H$ is the paw (the graph $\overline{P_{1}+P_{3}}$ ). In Section 7 we conclude our paper with some remarks for future work.

\section{Preliminaries}

Throughout the paper, we consider only finite, undirected graphs without multiple edges or self-loops. Below, we define further graph terminology.

The disjoint union $(V(G) \cup V(H), E(G) \cup E(H)$ ) of two vertex-disjoint graphs $G$ and $H$ is denoted by $G+H$ and the disjoint union of $r$ copies of a graph $G$ is denoted by $r G$. The complement $\bar{G}$ of a graph $G$ has vertex set $V(\bar{G})=V(G)$ and an edge between two distinct vertices $u, v$ if and only if $u v \notin E(G)$. For a subset $S \subseteq V(G)$, we let $G[S]$ denote the subgraph of $G$ induced by $S$, which has vertex set $S$ and edge set $\{u v \mid u, v \in S, u v \in E(G)\}$. If $S=\left\{s_{1}, \ldots, s_{r}\right\}$ then, to simplify notation, we may also write $G\left[s_{1}, \ldots, s_{r}\right]$ instead of $G\left[\left\{s_{1}, \ldots, s_{r}\right\}\right]$. We use $G \backslash S$ to denote the graph obtained from $G$ by deleting every vertex in $S$, that is, $G \backslash S=G[V(G) \backslash S]$. We write $G^{\prime} \subseteq_{i} G$ to indicate that $G^{\prime}$ is an induced subgraph of $G$.

The graphs $C_{r}, K_{r}, K_{1, r-1}$ and $P_{r}$ denote the cycle, complete graph, star and path on $r$ vertices, respectively. The graphs $K_{3}$ and $K_{1,3}$ are also called the triangle and claw, respectively. A graph $G$ is a linear forest if every component of $G$ is a path (on at least one vertex). The graph $S_{h, i, j}$, for $1 \leq h \leq i \leq j$, denotes the subdivided claw, that is, the tree that has only one vertex $x$ of degree 3 and exactly three leaves, which are at distance $h, i$ and $j$ from $x$, respectively. Observe that $S_{1,1,1}=K_{1,3}$. We let $\mathcal{S}$ denote the class of graphs, each connected component of which is either a subdivided claw or a path.

For a set of graphs $\left\{H_{1}, \ldots, H_{p}\right\}$, a graph $G$ is $\left(H_{1}, \ldots, H_{p}\right)$-free if it has no induced subgraph isomorphic to a graph in $\left\{H_{1}, \ldots, H_{p}\right\}$; if $p=1$, we may write $H_{1}$-free instead of $\left(H_{1}\right)$-free. A graph is $k$-partite if its vertex can be partitioned into $k$ (possibly empty) independent sets; 2-partite graphs are also known as bipartite graphs. The biclique or complete bipartite graph $K_{r, s}$ is the bipartite graph with sets in the partition of size $r$ and $s$ respectively, such that every vertex in one set is adjacent to every vertex in the other set. For a graph $G=(V, E)$, the set $N(u)=\{v \in V \mid u v \in E\}$ denotes the neighbourhood of $u \in V$. Let $X$ be a set of vertices in $G$. A vertex $y \in V \backslash X$ is complete to $X$ if it is adjacent to every vertex of $X$ and anti-complete to $X$ if it is non-adjacent to every vertex of $X$. A set of vertices $Y \subseteq V \backslash X$ is complete (resp. anti-complete) to $X$ if every vertex in $Y$ is complete (resp. anti-complete) to $X$. If $X$ and $Y$ are disjoint sets of vertices in a graph, we say that the edges between these two sets form a matching if each vertex in $X$ has at most one neighbour in $Y$ and vice versa; if each vertex has exactly one such neighbour, we say that the matching is perfect. Similarly, the edges between these sets form a co-matching if each vertex in $X$ has at most one non-neighbour in $Y$ and vice versa. We say that the set $X$ dominates $Y$ if every vertex of $Y$ has at least one neighbour in $X$. Similarly, a vertex $x$ dominates $Y$ if every vertex of $Y$ is adjacent to $x$. A vertex $y \in V \backslash X$ distinguishes $X$ if $y$ has both a neighbour and a non-neighbour in $X$. The set $X$ is a module of $G$ if no vertex in $V \backslash X$ distinguishes $X$. A module $X$ is non-trivial if $1<|X|<|V|$, otherwise it is trivial. A graph is prime if it has only trivial modules. Two vertices are false twins if they have the same neighbourhood (note that such vertices must be non-adjacent). Clearly any prime graph on at least three vertices cannot contain a pair of false twins, as any such pair of vertices would form a non-trivial module.

We will use the following structural result.

Lemma 1 ([12]). Let $G$ be a connected $\left(K_{3}, C_{5}, S_{1,2,3}\right)$-free graph that does not contain a pair of false twins. Then $G$ is either bipartite or a cycle.

\subsection{Clique-width}

The clique-width $\operatorname{cw}(G)$ of a graph $G$ is the minimum number of labels needed to construct $G$ by using the following four operations:

1. $i(v)$ : creating a new graph consisting of a single vertex $v$ with label $i$;

2. $G_{1} \oplus G_{2}$ : taking the disjoint union of two labelled graphs $G_{1}$ and $G_{2}$;

3. $\eta_{i, j}$ : joining each vertex with label $i$ to each vertex with label $j(i \neq j)$;

4. $\rho_{i \rightarrow j}$ : renaming label $i$ to $j$. 
We say that a construction of a graph $G$ with the four operations is a $k$-expression if it uses at most $k$ labels. Thus the clique-width of $G$ is the minimum $k$ for which $G$ has a $k$-expression. Recall that a class of graphs $\mathcal{G}$ has bounded clique-width if there is a constant $c$ such that the clique-width of every graph in $\mathcal{G}$ is at most $c$; otherwise the clique-width is unbounded.

Let $G$ be a graph. We define the following operations. For an induced subgraph $G^{\prime} \subseteq_{i} G$, the subgraph complementation operation (acting on $G$ with respect to $G^{\prime}$ ) replaces every edge present in $G^{\prime}$ by a non-edge, and vice versa. Similarly, for two disjoint vertex subsets $S$ and $T$ in $G$, the bipartite complementation operation with respect to $S$ and $T$ acts on $G$ by replacing every edge with one end-vertex in $S$ and the other one in $T$ by a non-edge and vice versa.

We now state some useful facts about how the above operations (and some other ones) influence the clique-width of a graph. We will use these facts throughout the paper. Let $k \geq 0$ be a constant and let $\gamma$ be some graph operation. We say that a graph class $\mathcal{G}^{\prime}$ is $(k, \gamma)$-obtained from a graph class $\mathcal{G}$ if the following two conditions hold:

1. every graph in $\mathcal{G}^{\prime}$ is obtained from a graph in $\mathcal{G}$ by performing $\gamma$ at most $k$ times, and

2. for every $G \in \mathcal{G}$ there exists at least one graph in $\mathcal{G}^{\prime}$ obtained from $G$ by performing $\gamma$ at most $k$ times.

We say that $\gamma$ preserves boundedness of clique-width if for any finite constant $k$ and any graph class $\mathcal{G}$, any graph class $\mathcal{G}^{\prime}$ that is $(k, \gamma)$-obtained from $\mathcal{G}$ has bounded clique-width if and only if $\mathcal{G}$ has bounded clique-width.

Fact 1. Vertex deletion preserves boundedness of clique-width [36].

Fact 2. Subgraph complementation preserves boundedness of clique-width [29].

Fact 3. Bipartite complementation preserves boundedness of clique-width [29].

Lemma 2 ([11]). Let $G$ be a graph and let $\mathcal{P}$ be the set of all induced subgraphs of $G$ that are prime. Then $\mathrm{cw}(G)=\max _{H \in \mathcal{P}} \mathrm{cw}(H)$.

\subsection{Well-quasi-orderability}

A quasi order $\leq$ on a set $X$ is a reflexive, transitive binary relation. Two elements $x, y \in X$ in this quasi-order are comparable if $x \leq y$ or $y \leq x$, otherwise they are incomparable. A set of elements in a quasi-order is a chain if every pair of elements is comparable and it is an antichain if every pair of elements is incomparable. The quasi-order $\leq$ is a well-quasiorder if any infinite sequence of elements $x_{1}, x_{2}, x_{3}, \ldots$ in $X$ contains a pair $\left(x_{i}, x_{j}\right)$ with $x_{i} \leq x_{j}$ and $i<j$. Equivalently, a quasi-order is a well-quasi-order if and only if it has no infinite strictly decreasing sequence $x_{1} \geqslant x_{2} \geqslant x_{3} \geqslant \cdots$ and no infinite antichain.

For an arbitrary set $M$, let $M^{*}$ denote the set of finite sequences of elements of $M$. A quasi-order $\leq$ on $M$ defines a quasi-order $\leq^{*}$ on $M^{*}$ as follows: $\left(a_{1}, \ldots, a_{m}\right) \leq^{*}\left(b_{1}, \ldots, b_{n}\right)$ if and only if there is a sequence of integers $i_{1}, \ldots, i_{m}$ with $1 \leq i_{1}<\cdots<i_{m} \leq n$ such that $a_{j} \leq b_{i_{j}}$ for $j \in\{1, \ldots, m\}$. We call $\leq^{*}$ the subsequence relation.

Lemma 3 (Higman's Lemma [27]). If $(M, \leq)$ is a well-quasi-order then $\left(M^{*}, \leq^{*}\right)$ is a well-quasi-order.

To define the notion of labelled induced subgraphs, let us consider an arbitrary quasi-order $(W, \leq)$. We say that $G$ is a labelled graph if each vertex $v$ of $G$ is equipped with an element $l_{G}(v) \in W$ (the label of $v$ ). Given two labelled graphs $G$ and $H$, we say that $G$ is a labelled induced subgraph of $H$ if $G$ is isomorphic to an induced subgraph of $H$ and there is an isomorphism that maps each vertex $v$ of $G$ to a vertex $w$ of $H$ with $l_{G}(v) \leq l_{H}(w)$. Clearly, if $(W, \leq)$ is a well-quasi-order, then a graph class $X$ cannot contain an infinite sequence of labelled graphs that is strictly-decreasing with respect to the labelled induced subgraph relation. We therefore say that a graph class $X$ is well-quasi-ordered by the labelled induced subgraph relation if it contains no infinite antichains of labelled graphs whenever $(W, \leq)$ is a well-quasi-order. Such a class is readily seen to also be well-quasi-ordered by the induced subgraph relation.

Daligault, Rao and Thomassé [18] showed that every hereditary class of graphs that is well-quasi-ordered by the labelled induced subgraph relation is defined by a finite set of forbidden induced subgraphs. Korpelainen, Lozin and Razgon [34] conjectured that if a hereditary class of graphs $\mathcal{G}$ is defined by a finite set of forbidden induced subgraphs, then $\mathcal{G}$ is wellquasi-ordered by the induced subgraph relation if and only if it is well-quasi-ordered by the labelled induced subgraph relation. Brignall, Engen and Vatter [8] recently found a class $\mathcal{G}^{*}$ with 14 forbidden induced subgraphs that is a counterexample for this conjecture, that is $\mathcal{G}^{*}$ is well-quasi-ordered by the induced subgraph relation but not by the labelled induced subgraph relation. However, so far, all known results for bigenic graph classes, including those in this paper, agree with the conjecture for bigenic graph classes.

Similarly to the notion of preserving boundedness of clique-width, we say that a graph operation $\gamma$ preserves well-quasiorderability by the labelled induced subgraph relation if for any finite constant $k$ and any graph class $\mathcal{G}$, any graph class $\mathcal{G}^{\prime}$ that is $(k, \gamma)$-obtained from $\mathcal{G}$ is well-quasi-ordered by this relation if and only if $\mathcal{G}$ is. 
Lemma 4 ([15]). The following operations preserve well-quasi-orderability by the labelled induced subgraph relation:

(i) Subgraph complementation,

(ii) Bipartite complementation and

(iii) Vertex deletion.

Lemma 5 ([3]). A hereditary class $X$ of graphs is well-quasi-ordered by the labelled induced subgraph relation if and only if the set of prime graphs in $X$ is. In particular, $X$ is well-quasi-ordered by the labelled induced subgraph relation if and only if the set of connected graphs in $X$ is.

Lemma 6 ([3,32]). ( $\left.P_{7}, S_{1,2,3}\right)$-free bipartite graphs are well-quasi-ordered by the labelled induced subgraph relation.

Let $\left(L_{1}, \leq_{1}\right)$ and $\left(L_{2}, \leq_{2}\right)$ be well-quasi-orders. We define the Cartesian Product $\left(L_{1}, \leq_{1}\right) \times\left(L_{2}\right.$, $\left.\leq_{2}\right)$ of these well-quasiorders as the order $\left(L, \leq_{L}\right)$ on the set $L:=L_{1} \times L_{2}$ where $\left(l_{1}, l_{2}\right) \leq_{L}\left(l_{1}^{\prime}, l_{2}^{\prime}\right)$ if and only if $l_{1} \leq_{1} l_{1}^{\prime}$ and $l_{2} \leq_{2} l_{2}^{\prime}$. Lemma 3 implies that $\left(L, \leq_{L}\right)$ is also a well-quasi-order. If $G$ has a labelling with elements of $L_{1}$ and of $L_{2}$, say $l_{1}: V(G) \rightarrow L_{1}$ and $l_{2}: V(G) \rightarrow L_{2}$, we can construct the combined labelling in $\left(L_{1}, \leq_{1}\right) \times\left(L_{2}, \leq_{2}\right)$ that labels each vertex $v$ of $G$ with the label $\left(l_{1}(v), l_{2}(v)\right)$.

Lemma 7. Fix a well-quasi-order $\left(L_{1}, \leq_{1}\right)$ that has at least one element. Let $X$ be a class of graphs. For each $G \in X$ fix a labelling $l_{G}^{1}$ : $V(G) \rightarrow L_{1}$. Then $X$ is well-quasi-ordered by the labelled induced subgraph relation if and only if for every well-quasi-order $\left(L_{2}, \leq_{2}\right)$ and every labelling of the graphs in $X$ by this order, the combined labelling in $\left(L_{1}, \leq_{1}\right) \times\left(L_{2}, \leq_{2}\right)$ obtained from these labellings also results in a well-quasi-ordered set of labelled graphs.

Proof. If $X$ is well-quasi-ordered by the labelled induced subgraph relation then by definition it is well-quasi-ordered when labelled with labels from $\left(L_{1}, \leq_{1}\right) \times\left(L_{2}, \leq_{2}\right)$. If $X$ is not well-quasi-ordered by the labelled induced subgraph relation then there must be a well-quasi-order $\left(L_{2}, \leq_{2}\right)$ and an infinite set of graphs $G_{1}, G_{2}, \ldots$ whose vertices are labelled with elements of $L_{2}$ such that these graphs form an infinite labelled antichain. For each graph $G_{i}$, replace the label $l$ on vertex $v$ by $\left(l_{G_{1}}^{1}(v), l\right)$. The graphs are now labelled with elements of the well-quasi-order $\left(L_{1}, \leq_{1}\right) \times\left(L_{2}, \leq_{2}\right)$ and result in an infinite labelled antichain of graphs labelled with such combined labellings. This completes the proof.

\section{3. $k$-uniform graphs}

For an integer $k \geq 1$, a graph $G$ is $k$-uniform if there is a symmetric square 0,1 matrix $K$ of order $k$ and a graph $F_{k}$ on vertices $1,2, \ldots, k$ such that $G \in \mathcal{P}\left(K, F_{k}\right)$, where $\mathcal{P}\left(K, F_{k}\right)$ is the graph class defined as follows. Let $H$ be the disjoint union of infinitely many copies of $F_{k}$. For $i \in\{1, \ldots, k\}$, let $V_{i}$ be the subset of $V(H)$ containing vertex $i$ from each copy of $F_{k}$. Construct from $H$ an infinite graph $H(K)$ on the same vertex set by applying a subgraph complementation to $V_{i}$ if and only if $K(i, i)=1$ and by applying a bipartite complementation to a pair $V_{i}, V_{j}$ if and only if $K(i, j)=1$. Thus, two vertices $u \in V_{i}$ and $v \in V_{j}$ are adjacent in $H(K)$ if and only if $u v \in E(H)$ and $K(i, j)=0$ or $u v \notin E(H)$ and $K(i, j)=1$. Then, $\mathcal{P}\left(K, F_{k}\right)$ is the hereditary class consisting of all the finite induced subgraphs of $H(K)$. The minimum $k$ such that $G$ is $k$-uniform is the uniformicity of $G$. The second of the next two lemmas follows directly from the above definitions.

The following result was proved by Korpelainen and Lozin. The class of disjoint unions of cliques is a counterexample for the reverse implication.

Lemma 8 ([33]). Any class of graphs of bounded uniformicity is well-quasi-ordered by the labelled induced subgraph relation.

The following lemma follows from the definition of clique-width and the definition of $k$-uniform graphs (see also [2] for a more general result). For completeness, we include a formal proof.

Lemma 9. For every $k \geq 1$, every $k$-uniform graph has clique-width at most $2 k$.

Proof. Let $k \geq 1$ and let $H(K)$ be the graph from the definition of $k$-uniform for some $F_{k}$ and $K$. We will show that every finite induced subgraph of $H(K)$ has clique-width at most $2 k$. Let $X_{1}, X_{2}, \ldots$ be the sets of vertices corresponding to each copy of $F_{k}$ in the construction of $H(K)$ and note that $\left|X_{h}\right|=k$ for all $h \geq 1$ and that $\left|X_{h} \cap V_{i}\right|=1$ for all $h \geq 1, i \in\{1, \ldots, k\}$. Furthermore, if two vertices $x \in V_{i}$ and $y \in V_{j}$ do not lie in the same set $X_{h}$, then $x$ and $y$ are adjacent if and only if $K(i, j)=1$. For $\ell \geq 0$, let $H_{\ell}=H(K)\left[X_{1} \cup \cdots \cup X_{\ell}\right]$ (so $H_{0}$ is the empty graph, which has no vertices).

We will prove by induction on $\ell \geq 0$ that there is a $2 k$-expression for $H_{\ell}$, with the property that once this $2 k$-expression has been applied, for all $i \in\{1, \ldots, k\}$, every vertex in $V\left(H_{\ell}\right) \cap V_{i}$ has label $i$. The $\ell=0$ case is trivial. Now suppose that the induction hypothesis holds for some $\ell \geq 0$, so there is a $2 k$-expression for $H_{\ell}$ with the property that once this $2 k$-expression has been applied, for all $i \in\{1, \ldots, k\}$ every vertex in $V\left(H_{\ell}\right) \cap V_{i}$ has label $i$. We will construct a $2 k$-expression for $H_{\ell+1}$ 


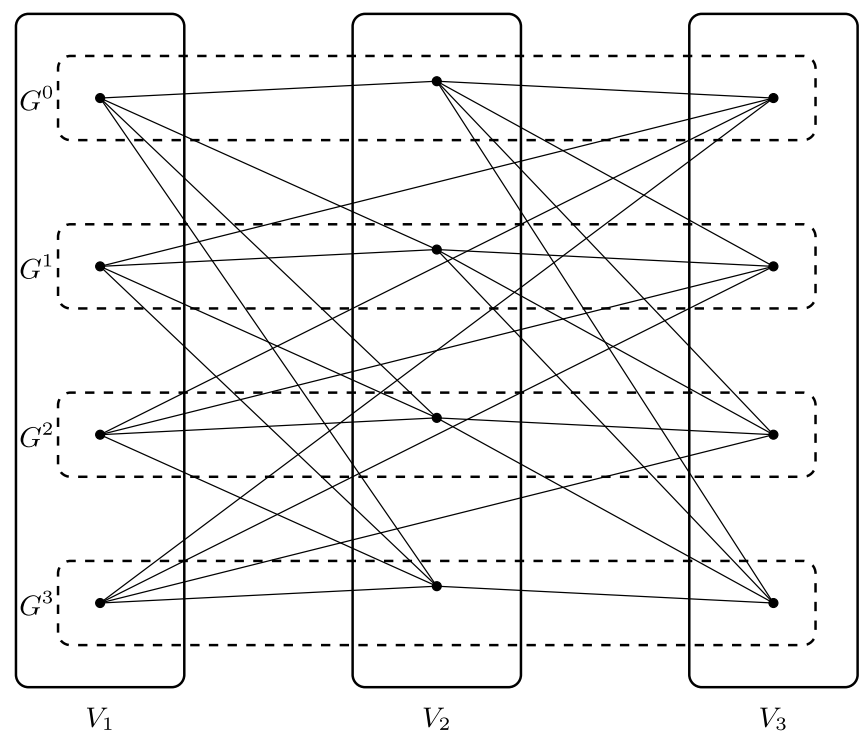

Fig. 2. A 3-partite graph partitioned into slices $G^{0}, \ldots, G^{3}$ isomorphic to $P_{3}$.

with the property that once this new $2 k$-expression has been applied, for all $i \in\{1, \ldots, k\}$ every vertex in $V\left(H_{\ell+1}\right) \cap V_{i}$ has label $i$. To do this, we first take the $2 k$-expression for $H_{\ell}$ and then for every $i \in\{1, \ldots, k\}$, we create the unique vertex of $X_{\ell+1} \cap V_{i}$ with label $k+i$ and take the disjoint union of these vertices with the constructed $H_{\ell}$. Next, for $i, j \in\{1, \ldots, k\}$ with $i<j$, if the unique vertex of $X_{\ell+1} \cap V_{i}$ is adjacent to the unique vertex of $X_{\ell+1} \cap V_{j}$ in $H_{\ell+1}$, then we apply the join operation $\eta_{k+i, k+j}()$. Next, for $i, j \in\{1, \ldots, k\}$, if $K(i, j)=1$, then we apply the join operation $\eta_{i, k+j}()$. Finally, for every $i \in\{1, \ldots, k\}$ we apply the relabel operation $\rho_{k+i \rightarrow i}()$. The resulting $2 k$-expression constructs $H_{\ell+1}$ with the required labels, so the induction hypothesis also holds for $\ell+1$. By induction, the induction hypothesis holds for every $\ell \geq 0$ and so we conclude that $H_{\ell}$ has clique-width at most $2 k$ for every $\ell$.

Now if $G$ is a $k$-uniform graph then it is a finite induced subgraph of $H(K)$, so there is an $\ell$ such that $G$ is an induced subgraph of $H_{\ell}$. It follows that $G$ has clique-width at most $2 k$. This completes the proof.

\section{Outline of our proof method}

We prove our results on both well-quasi-orderability and boundedness of clique-width by a further generalization of the canonical decomposition method introduced by Fouquet, Giakoumakis and Vanherpe [24] for bipartite graphs. As discussed in Section 1, this method was generalized to 3-partite graphs in [12], but for our purposes we need a non-trivial refinement. We explain this refinement in detail in Section 4, but below we sketch the main ideas behind it. In order to do this, we first need to introduce some terminology.

Let $G$ be a 3-partite graph given with a partition of its vertex set into three independent sets $V_{1}, V_{2}$ and $V_{3}$. Suppose that each set $V_{i}$ can be partitioned into sets $V_{i}^{0}, \ldots, V_{i}^{\ell}$ (the value of $\ell$ is the same for each $i \in\{1,2,3\}$ ) such that, taking subscripts modulo 3:

- for $i \in\{1,2,3\}$ if $j<k$, then $V_{i}^{j}$ is complete to $V_{i+1}^{k}$ and anti-complete to $V_{i+2}^{k}$.

For $i \in\{0, \ldots, \ell\}$ let $G^{i}=G\left[V_{1}^{i} \cup V_{2}^{i} \cup V_{3}^{i}\right]$. We say that the graphs $G^{i}$ are the slices of $G$. If the slices belong to some graph class $X$, then we say that $G$ can be partitioned into slices from $X$; see Fig. 2 for an example.

In Section 4.1 we prove the following two results:

(i) every 3-partite graph that can be partitioned into slices of clique-width at most $k$ has clique-width at most max(3k,6);

(ii) for every set $Y$ of 3-partite graphs that belong to some hereditary graph class $X$ : if the graphs in $Y$ can be partitioned into slices from some subclass $Z \subseteq X$ that is well-quasi-ordered by the labelled induced subgraph relation, then $Y$ is also well-quasi-ordered by the labelled induced subgraph relation.

In Section 4.2, we give sufficient conditions for a 3-partite graph to have the above decomposition into slices, so that we can apply results (i) and (ii) for boundedness of clique-width and being well-quasi-ordered by the labelled induced subgraph relation (and thus by the induced subgraph relation), respectively. To explain these conditions, we need to introduce some more terminology. Let $G$ be a 3-partite graph given together with a partition of its vertex set into three independent sets $V_{1}, V_{2}$ and $V_{3}$. An induced $K_{3}$ or $3 P_{1}$ in $G$ is rainbow if it has exactly one vertex in each set $V_{i}$. We say that $G$ is curious 
with respect to the partition $\left(V_{1}, V_{2}, V_{3}\right)$ if it contains no rainbow $K_{3}$ or $3 P_{1}$ when its vertex set is partitioned in this way. We say that $G$ is curious if there is a partition $\left(V_{1}, V_{2}, V_{3}\right)$ with respect to which it is curious; note that bipartite graphs are curious, since we allow the case when $V_{i}=\emptyset$ for some $i$. In Section 4.2 we will prove the following result:

(iii) if the set $X^{\prime}$ of bipartite graphs in a hereditary graph class $X$ is well-quasi-ordered by the labelled induced subgraph relation or have bounded clique-width, then the same is true for the curious graphs in $X$.

We will prove result (iii) by showing that the curious graphs in $X$ allow a partition into slices from $X^{\prime}$, so that we can indeed apply results (i) and (ii).

Finally, in Section 5, we apply result (iii) on the classes of $\left(K_{3}, P_{1}+P_{5}\right)$-free graphs and $\left(K_{3}, P_{2}+P_{4}\right)$-free graphs, in order to prove that these classes have bounded clique-width and are well-quasi-ordered by the labelled induced subgraph relation. To do this, we prove two structural lemmas. These lemmas consider the case when a graph in one of these classes contains an induced subgraph isomorphic to $C_{5}$. We partition the remaining vertices of the graph into sets according to their neighbourhood in this $C_{5}$ and analyse the edges between the vertices in these sets. We use this analysis to show how to use a constant number of vertex deletions and bipartite complementations to transform the graph into a disjoint union of curious graphs and 3-uniform graphs in the respective graph class. The two lemmas enable us to apply result (iii) after combining them with Theorem 3 and Lemma 6, respectively, which imply that the bipartite graphs in the classes of $\left(K_{3}, P_{1}+P_{5}\right)$-free graphs and $\left(K_{3}, P_{2}+P_{4}\right)$-free graphs have bounded clique-width and are well-quasi-ordered by the (labelled) induced subgraph relation, respectively.

\section{Partitioning 3-partite graphs}

In Section 4.1 we recall our graph decomposition on 3-partite graphs. We then show how to extend results on bounded clique-width or well-quasi-orderability by the labelled induced subgraph relation from bipartite graphs in an arbitrary hereditary class of graphs to the 3-partite graphs in this class that are decomposable in this way. In Section 4.2 we give sufficient conditions for a 3-partite graph to have such a decomposition.

\subsection{The decomposition}

Let $G$ be a 3-partite graph given with a partition of its vertex set into three independent sets $V_{1}, V_{2}$ and $V_{3}$. Suppose that each set $V_{i}$ can be partitioned into sets $V_{i}^{0}, \ldots, V_{i}^{\ell}$ (the value of $\ell$ is the same for each $\left.i \in\{1,2,3\}\right)$ such that, taking subscripts modulo 3 :

- for $i \in\{1,2,3\}$ if $j<k$ then $V_{i}^{j}$ is complete to $V_{i+1}^{k}$ and anti-complete to $V_{i+2}^{k}$.

For $i \in\{0, \ldots, \ell\}$ let $G^{i}=G\left[V_{1}^{i} \cup V_{2}^{i} \cup V_{3}^{i}\right]$. Recall that the graphs $G^{i}$ are the slices of $G$ and that if the slices belong to some graph class $X$, then $G$ can be partitioned into slices from $X$.

Lemma 10. If $G$ is a 3-partite graph that can be partitioned into slices of clique-width at most $k$, then $G$ has clique-width at most $\max (3 k, 6)$.

Proof. Since every slice $G^{j}$ of $G$ has clique-width at most $k$, it can be constructed using a $k$-expression, which uses the labels $1, \ldots, k$. Applying relabelling operations if necessary, we may assume that at the end of this construction, every vertex has label 1 . We can modify this $k$-expression so that we use the $3 k$ labels $1_{1}, \ldots, k_{1}, 1_{2}, \ldots, k_{2}, 1_{3}, \ldots, k_{3}$ instead (so the modified expression will be a $3 k$-expression for $G^{j}$ ), in such a way that at all points in the construction, for each $i \in\{1,2,3\}$ every constructed vertex in $V_{i}$ has a label in $\left\{1_{i}, \ldots, k_{i}\right\}$. To do this we replace:

- creation operations $i(v)$ by $i_{j}(v)$ if $v \in V_{j}$,

- relabel operations $\rho_{j \rightarrow k}()$ by $\rho_{j_{1} \rightarrow k_{1}}\left(\rho_{j_{2} \rightarrow k_{2}}\left(\rho_{j_{3} \rightarrow k_{3}}()\right)\right)$ and

- join operations $\eta_{j, k}()$ by

$$
\eta_{j_{1}, k_{1}}\left(\eta_{j_{1}, k_{2}}\left(\eta_{j_{1}, k_{3}}\left(\eta_{j_{2}, k_{1}}\left(\eta_{j_{2}, k_{2}}\left(\eta_{j_{2}, k_{3}}\left(\eta_{j_{3}, k_{1}}\left(\eta_{j_{3}, k_{2}}\left(\eta_{j_{3}, k_{3}}()\right)\right)\right)\right)\right)\right)\right)\right) \text {. }
$$

This new $3 k$-expression uses $3 k$ labels and at the end of it, every vertex in $V_{i}$ is labelled with label $1_{i}$. We find such a $3 k$-expression $E^{j}$ for every slice $G^{j}$ of $G$ independently.

We now prove by induction on $j \geq 0$ that there is a $\max (3 k, 6)$-expression which constructs $G\left[V\left(G^{0}\right) \cup \cdots \cup V\left(G^{j}\right)\right]$ in such a way that every vertex in $V_{i}$ is labelled with label $2_{i}$. For $j=0$ we simply take the $3 k$-expression $E^{0}$ for $G^{0}$ and then apply three relabelling operations $\rho_{1_{1} \rightarrow 2_{1}}, \rho_{1_{2} \rightarrow 2_{2}}$ and $\rho_{1_{3} \rightarrow 2_{3}}$. Suppose the induction hypothesis holds for some $j \geq 0$ and let $F^{j}$ be the corresponding $\max (3 k, 6)$-expression for $G\left[V\left(G^{0}\right) \cup \cdots \cup V\left(G^{j}\right)\right]$. We take the disjoint union of the $\max (3 k, 6)$-expressions $F^{j}$ and $E^{j+1}$. Next, we apply join operations $\eta_{2_{1}, 1_{2}}, \eta_{2_{2}, 1_{3}}$ and $\eta_{2_{3}, 1_{1}}$. Finally, we apply the 
relabelling operations $\rho_{1_{1} \rightarrow 2_{1}}, \rho_{1_{2} \rightarrow 2_{2}}$ and $\rho_{1_{3} \rightarrow 2_{3}}$. This yields a max(3k,6)-expression for $G\left[V\left(G^{0}\right) \cup \cdots \cup V\left(G^{j+1}\right)\right]$ in such a way that every vertex in $V_{i}$ is labelled with $2_{i}$. (Note that in the obtained expression we only ever use the labels $\left\{1_{1}, \ldots, \max (k, 2)_{1}, 1_{2}, \ldots, \max (k, 2)_{2}, 1_{3}, \ldots, \max (k, 2)_{3}\right\}$, so it is indeed a $\max (3 k, 6)$-expression.) Therefore the induction hypothesis holds for every $j \geq 0$. By induction, it follows that $G$ has clique-width at most $\max (3 k, 6)$.

Lemma 11. Let $X$ be a hereditary graph class containing a class $Z$. Let $Y$ be the set of 3-partite graphs in $X$ that can be partitioned into slices from $Z$. If $Z$ is well-quasi-ordered by the labelled induced subgraph relation, then so is $Y$.

Proof. For each graph $G$ in $Y$, we may fix a partition into independent sets $\left(V_{1}, V_{2}, V_{3}\right)$ with respect to which the graph can be partitioned into slices from $Z$. Let $\left(L_{1}, \leq_{1}\right)$ be the well-quasi-order with $L_{1}=\{1,2,3\}$ in which every pair of distinct elements is incomparable. By Lemma 7, we need only consider labellings of graphs in $Y$ of the form $(i, l(v))$ where $v \in V_{i}$ and $l(v)$ belongs to an arbitrary well-quasi-order $L$. Suppose $G$ can be partitioned into slices $G^{0}, \ldots, G^{k}$, with vertices labelled as in $G$. Note that the ordered list $G^{0}, \ldots, G^{k}$ completely specifies the edges of $G$ and the labels on its vertices. Indeed, two vertices $x, y \in V\left(G^{i}\right)$ are adjacent in $G$ if and only if they are adjacent in $G^{i}$ and two vertices $x \in V\left(G^{i}\right)$, $y \in V\left(G^{j}\right)$ with $i<j$ are adjacent in $G$ if and only if $x \in V_{k}$ and $y \in V_{k+1}$ for some $k \in\{1,2,3\}$ (subscripts taken modulo 3 ).

Now consider two labelled graphs $G, H \in Y$ with corresponding partitions into slices $G^{0}, \ldots, G^{k}$ and $H^{0}, \ldots, H^{\ell}$. If $\left(H^{0}, \ldots, H^{\ell}\right)$ is smaller than or equal to $\left(G^{0}, \ldots, G^{k}\right)$ under the subsequence relation, then $H$ is a labelled induced subgraph of $G$. The result follows by Lemma 3.

\subsection{Curious graphs}

Let $G$ be a 3-partite graph given together with a partition of its vertex set into three independent sets $V_{1}, V_{2}$ and $V_{3}$. Recall that an induced $K_{3}$ or $3 P_{1}$ in $G$ is rainbow if it has exactly one vertex in each set $V_{i}$. Also recall that $G$ is curious with respect to the partition $\left(V_{1}, V_{2}, V_{3}\right)$ if it contains no rainbow $K_{3}$ or $3 P_{1}$ when its vertex set is partitioned in this way, and that $G$ is curious if there is a partition $\left(V_{1}, V_{2}, V_{3}\right)$ with respect to which it is curious. In this section we will prove that given a hereditary class $X$, if the bipartite graphs in $X$ are well-quasi-ordered by the labelled induced subgraph relation or have bounded clique-width, then the same is true for the curious graphs in $X$.

A linear order $\left(x_{1}, x_{2}, \ldots, x_{k}\right)$ of the vertices of an independent set $I$ is

- increasing if $i<j$ implies $N\left(x_{i}\right) \subseteq N\left(x_{j}\right)$,

- decreasing if $i<j$ implies $N\left(x_{i}\right) \supseteq N\left(x_{j}\right)$,

- monotone if it is either increasing or decreasing.

Bipartite graphs that are $2 P_{2}$-free are also known as bipartite chain graphs. It is well known (and easy to verify) that a bipartite graph $G$ is $2 P_{2}$-free if and only if the vertices in each independent set of the bipartition admit a monotone ordering. Suppose $G$ is a curious graph with respect to some partition $\left(V_{1}, V_{2}, V_{3}\right)$. We say that (with respect to this partition) the graph $G$ is a curious graph of type $t$ if exactly $t$ of the graphs $G\left[V_{1} \cup V_{2}\right], G\left[V_{1} \cup V_{3}\right]$ and $G\left[V_{2} \cup V_{3}\right]$ contain an induced $2 P_{2}$.

\subsubsection{Curious graphs of type 0 and 1}

Note that if $G$ is a curious graph of type 0 or 1 with respect to the partition $\left(V_{1}, V_{2}, V_{3}\right)$ then without loss of generality, we may assume that $G\left[V_{1} \cup V_{2}\right]$ and $G\left[V_{1} \cup V_{3}\right]$ are both $2 P_{2}$-free.

Lemma 12. Let $G$ be a curious graph with respect to $\left(V_{1}, V_{2}, V_{3}\right)$, such that $G\left[V_{1} \cup V_{2}\right]$ and $G\left[V_{1} \cup V_{3}\right]$ are both $2 P_{2}$-free. Then the vertices of $V_{1}$ admit a linear ordering which is decreasing in $G\left[V_{1} \cup V_{2}\right]$ and increasing in $G\left[V_{1} \cup V_{3}\right]$.

Proof. For a set $S \subseteq V$, we use $N_{S}(u):=N(u) \cap S$ to denote the set of vertices in $S$ that are adjacent to $u$. We may choose a linear order $x_{1}, \ldots, x_{\ell}$ of the vertices of $V_{1}$ according to their neighbourhood in $V_{2}$, breaking ties according to their neighbourhood in $V_{3}$ i.e. an order such that:

(i) if $N_{V_{2}}\left(x_{i}\right) \supsetneq N_{V_{2}}\left(x_{j}\right)$ then $i<j$ and

(ii) if $N_{V_{2}}\left(x_{i}\right)=N_{V_{2}}\left(x_{j}\right)$ and $N_{V_{3}}\left(x_{i}\right) \subsetneq N_{V_{3}}\left(x_{j}\right)$ then $i<j$.

Clearly such an ordering is decreasing in $G\left[V_{1} \cup V_{2}\right]$.

Suppose, for contradiction, that this order is not increasing in $G\left[V_{1} \cup V_{3}\right]$. Then there must be indices $i<j$ such that $N_{V_{3}}\left(x_{i}\right) \supsetneq N_{V_{3}}\left(x_{j}\right)$. Then $N_{V_{2}}\left(x_{i}\right) \neq N_{V_{2}}\left(x_{j}\right)$ by Property (ii). By Property (i) it follows that $N_{V_{2}}\left(x_{i}\right) \supsetneq N_{V_{2}}\left(x_{j}\right)$. This means that there are vertices $y \in N_{V_{2}}\left(x_{i}\right) \backslash N_{V_{2}}\left(x_{j}\right)$ and $z \in N_{V_{3}}\left(x_{i}\right) \backslash N_{V_{3}}\left(x_{j}\right)$. Now if $y$ is adjacent to $z$ then $G\left[x_{i}, y, z\right]$ is a rainbow $K_{3}$ and if $y$ is non-adjacent to $z$ then $G\left[x_{j}, y, z\right]$ is a rainbow $3 P_{1}$. This contradiction implies that the order is indeed decreasing in $G\left[V_{1} \cup V_{3}\right]$, which completes the proof. 
Lemma 13. If $G$ is a curious graph of type 0 or 1 with respect to a partition $\left(V_{1}, V_{2}, V_{3}\right)$ then $G$ can be partitioned into slices that are bipartite.

Proof. Since $G$ is a curious graph of type 0 or 1 , without loss of generality, we may assume that $G\left[V_{1} \cup V_{2}\right]$ and $G\left[V_{1} \cup V_{3}\right]$ are both $2 P_{2}$-free. Let $\ell=\left|V_{1}\right|$ (we will partition $G$ into $\ell+1$ slices $G^{0}, \ldots, G^{\ell}$ ). Let $x_{1}, \ldots, x_{\ell}$ be a linear order on $V_{1}$ satisfying Lemma 12. Let $V_{1}^{0}=\emptyset$ and for $i \in\{1, \ldots, \ell\}$, let $V_{1}^{i}=\left\{x_{i}\right\}$. We partition $V_{2}$ and $V_{3}$ as follows. For $i \in\{0, \ldots, \ell\}$, let $V_{2}^{i}=\left\{y \in V_{2} \mid x_{j} y \in E(G)\right.$ if and only if $\left.j \leq i\right\}$. For $i \in\{0, \ldots, \ell\}$, let $V_{3}^{i}=\left\{z \in V_{3} \mid x_{j} z \notin E(G)\right.$ if and only if $\left.j \leq i\right\}$. In particular, note that the vertices of $V_{2}^{\ell} \cup V_{3}^{0}$ and $V_{2}^{0} \cup V_{3}^{\ell}$ are complete and anti-complete to $V_{1}$, respectively. The following properties hold:

- If $j<k$, then $V_{1}^{j}$ is complete to $V_{2}^{k}$ and anti-complete to $V_{3}^{k}$.

- If $j>k$, then $V_{1}^{j}$ is anti-complete to $V_{2}^{k}$ and complete to $V_{3}^{k}$.

If $j<k$ and $y \in V_{2}^{j}$ is non-adjacent to $z \in V_{3}^{k}$ then $G\left[x_{k}, y, z\right]$ is a rainbow $3 P_{1}$, a contradiction. If $j>k$ and $y \in V_{2}^{j}$ is adjacent to $z \in V_{3}^{k}$ then $G\left[x_{j}, y, z\right]$ is a rainbow $K_{3}$, a contradiction. It follows that:

- If $j<k$, then $V_{2}^{j}$ is complete to $V_{3}^{k}$.

- If $j>k$, then $V_{2}^{j}$ is anti-complete to $V_{3}^{k}$.

For $i \in\{0, \ldots, \ell\}$, let $G^{i}=G\left[V_{1}^{i} \cup V_{2}^{i} \cup V_{3}^{i}\right]$. The above properties about the edges between the sets $V_{j}^{i}$ imply the following statements:

- If $j<k$, then $V_{1}^{j}$ is complete to $V_{2}^{k}$ and anti-complete to $V_{3}^{k}$.

- If $j<k$, then $V_{2}^{j}$ is complete to $V_{3}^{k}$ and anti-complete to $V_{1}^{k}$.

- If $j<k$, then $V_{3}^{j}$ is complete to $V_{1}^{k}$ and anti-complete to $V_{2}^{k}$.

Thus $G$ can be partitioned into the slices $G^{0}, \ldots, G^{\ell}$. Now, for each $i \in\{0, \ldots, \ell\}, V_{1}^{i}$ is anti-complete to $V_{3}^{i}$, so every slice $G^{i}$ is bipartite. This completes the proof.

\subsubsection{Curious graphs of type 2 and 3}

Lemma 14. Fix $t \in\{2,3\}$. If $G$ is a curious graph of type $t$ with respect to a partition $\left(V_{1}, V_{2}, V_{3}\right)$ then $G$ can be partitioned into slices of type at most $t-1$.

Proof. Fix $t \in\{2,3\}$ and let $G$ be a curious graph of type $t$ with respect to a partition $\left(V_{1}, V_{2}, V_{3}\right)$. We may assume that $G\left[V_{1} \cup V_{2}\right]$ contains an induced $2 P_{2}$.

Claim 1. Given a $2 P_{2}$ in $G\left[V_{1} \cup V_{2}\right]$, every vertex of $V_{3}$ has exactly two neighbours in the $2 P_{2}$ and these neighbours either both lie in $V_{1}$ or both lie in $V_{2}$.

Let $x_{1}, x_{2} \in V_{1}$ and $y_{1}, y_{2} \in V_{2}$ induce a $2 P_{2}$ in $G$ such that $x_{1}$ is adjacent to $y_{1}$ but not to $y_{2}$ and $x_{2}$ is adjacent to $y_{2}$ but not to $y_{1}$. Consider $z \in V_{3}$. For $i \in\{1,2\}$, if $z$ is adjacent to both $x_{i}$ and $y_{i}$, then $G\left[x_{i}, y_{i}, z\right]$ is a rainbow $K_{3}$, a contradiction. Therefore $z$ can have at most one neighbour in $\left\{x_{1}, y_{1}\right\}$ and at most one neighbour in $\left\{x_{2}, y_{2}\right\}$. If $z$ is non-adjacent to $x_{1}$ and $y_{2}$ then $G\left[x_{1}, y_{2}, z\right]$ is a rainbow $3 P_{1}$, a contradiction. Therefore, $z$ can have at most one non-neighbour in $\left\{x_{1}, y_{2}\right\}$, and similarly $z$ can have at most one non-neighbour in $\left\{x_{2}, y_{1}\right\}$. Therefore, if $z$ is adjacent to $x_{1}$, then it must be non-adjacent to $y_{1}$, so it must be adjacent to $x_{2}$, so it must be non-adjacent to $y_{2}$. Similarly, if $z$ is non-adjacent to $x_{1}$ then it must be adjacent to $y_{2}$, so it must be non-adjacent to $x_{2}$, so it must be adjacent to $y_{1}$. Hence Claim 1 follows.

Consider a maximal set $\left\{H^{1}, \ldots, H^{q}\right\}$ of vertex-disjoint sets that induce copies of $2 P_{2}$ in $G\left[V_{1} \cup V_{2}\right]$. We say that a vertex of $V_{3}$ distinguishes two graphs $G\left[H^{i}\right]$ and $G\left[H^{j}\right]$ if its neighbours in $H^{i}$ and $H^{j}$ do not belong to the same set $V_{k}$. We group these sets $H^{i}$ into blocks $B^{1}, \ldots, B^{p}$ that are not distinguished by any vertex of $V_{3}$. In other words, every $B^{i}$ contains at least one $2 P_{2}$ and every vertex of $V_{3}$ is complete to one of the sets $B^{i} \cap V_{1}$ and $B^{i} \cap V_{2}$ and anti-complete to the other. For $j \in\{1,2\}$, let $B_{j}^{i}=B^{i} \cap V_{j}$. We define a relation $<_{B}$ on the blocks as follows:

- $B^{i}<_{B} B^{j}$ holds if $B_{1}^{i}$ is complete to $B_{2}^{j}$, while $B_{2}^{i}$ is anti-complete to $B_{1}^{j}$.

For distinct blocks $B^{i}, B^{j}$ at most one of $B^{i}<_{B} B^{j}$ and $B^{j}<_{B} B^{i}$ can hold. 


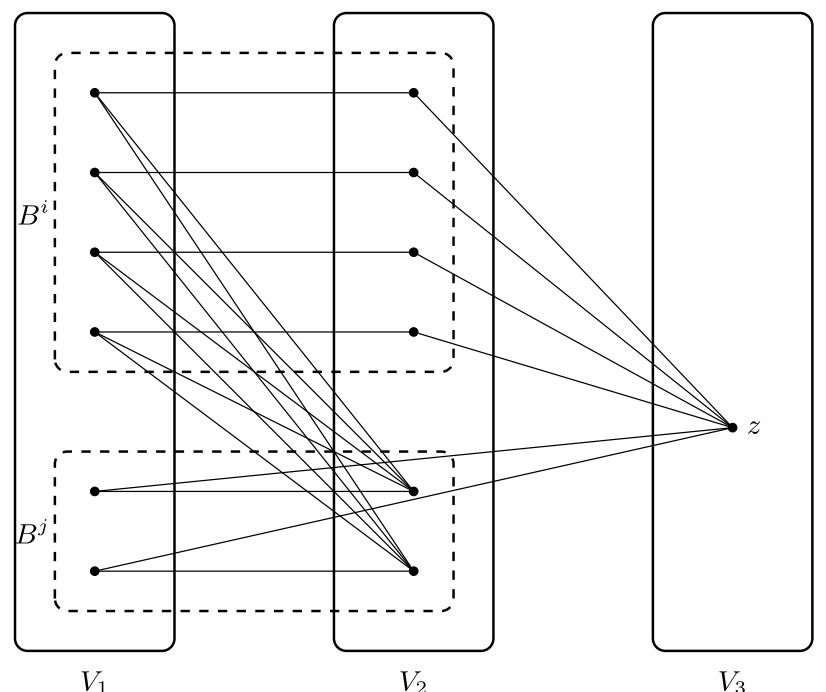

Fig. 3. Two blocks $B^{i}$ and $B^{j}$ with $B^{i}<_{B} B^{j}$ and a vertex $z \in V_{3}$ distinguishing them.

Claim 2. Let $B^{i}$ and $B^{j}$ be distinct blocks. There is a vertex $z \in V_{3}$ that distinguishes $B^{i}$ and $B^{j}$. If $z$ is complete to $B_{2}^{i} \cup B_{1}^{j}$ and anti-complete to $B_{1}^{i} \cup B_{2}^{j}$ then $B^{i}<_{B} B^{j}$ (see also Fig. 3). If $z$ is complete to $B_{2}^{j} \cup B_{1}^{i}$ and anti-complete to $B_{1}^{j} \cup B_{2}^{i}$ then $B^{j}<_{B} B^{i}$. Furthermore, one of these cases must apply, which implies that either $B^{i}<_{B} B^{j}$ or $B^{j}<_{B} B^{i}$ for every pair of distinct blocks $B^{i}$ and $B^{j}$.

By definition of the blocks $B^{i}$ and $B^{j}$ there must be a vertex $z \in V_{3}$ that distinguishes them. Without loss of generality we may assume that $z$ is complete to $B_{2}^{i} \cup B_{1}^{j}$ and anti-complete to $B_{1}^{i} \cup B_{2}^{j}$. It remains to show that $B^{i}<_{B} B^{j}$. If $y \in B_{2}^{i}$ is adjacent to $z \in B_{1}^{j}$ then $G[x, y, z]$ is a rainbow $K_{3}$, a contradiction. If $y \in B_{1}^{i}$ is non-adjacent to $z \in B_{2}^{j}$ then $G[x, y, z]$ is a rainbow $3 P_{1}$, a contradiction. Therefore $B_{2}^{i}$ is anti-complete to $B_{1}^{j}$ and $B_{1}^{i}$ is complete to $B_{2}^{j}$. It follows that $B^{i}<_{B} B^{j}$. Claim 2 follows by symmetry.

Claim 3. The relation $<_{B}$ is transitive.

Suppose that $B^{i}<_{B} B^{j}$ and $B^{j}<_{B} B^{k}$. Since $B^{j}$ and $B^{k}$ are distinct, there must be a vertex $z \in V_{3}$ that distinguishes them, and by combining Claim 2 with the fact that $B^{j}<_{B} B^{k}$ it follows that $z$ must be complete to $B_{2}^{j} \cup B_{1}^{k}$ and anti-complete to $B_{2}^{j} \cup B_{1}^{k}$. Suppose $x \in B_{1}^{i}$ and $y \in B_{2}^{j}$. Then $x$ is adjacent to $y$, since $B^{i}<_{B} B^{j}$ and $y$ is adjacent to $z$ by choice of $z$. Now $x$ is must be non-adjacent to $z$ otherwise $G[x, y, z]$ would be a rainbow $K_{3}$. Therefore $z$ is anti-complete to $B_{1}^{i}$. Recall that every vertex of $V_{3}$ is complete to one of $B_{1}^{i}$ and $B_{2}^{i}$ and anti-complete to the other (due to Claim 1). Therefore $z$ is complete to $B_{2}^{i}$, and so $z$ distinguishes $B^{i}$ and $B^{k}$. By Claim 2 it follows that $B^{i}<_{B} B^{k}$. This completes the proof of Claim 3.

Combining Claims $1-3$, we find that $<_{B}$ is a linear order on the blocks. We obtain the following conclusion, which we call the chain property.

Claim 4. The set of blocks admits a linear order $B^{1}<_{B} B^{2}<_{B} \cdots<_{B} B^{p}$ such that

(i) if $i<j$ then $B_{1}^{i}$ is complete to $B_{2}^{j}$, while $B_{2}^{i}$ is anti-complete to $B_{1}^{j}$ and

(ii) for each $z \in V_{3}$ there exists an $i \in\{0, \ldots, p\}$ such that if $j \leq i$ then $z$ is complete to $B_{2}^{j}$ and anti-complete to $B_{1}^{j}$ and if $j>i$ then $z$ is anti-complete to $B_{2}^{j}$ and complete to $B_{1}^{j}$.

Next consider the set of vertices in $V_{1} \cup V_{2}$ that do not belong to any set $B^{i}$. Let $R$ denote this set and note that $G[R]$ is $2 P_{2}$-free by maximality of the set $\left\{H^{1}, \ldots, H^{q}\right\}$. For $i \in\{1,2\}$ let $R_{i}=R \cap V_{i}$.

Claim 5. If $x \in R_{1}$ has a neighbour in $B_{2}^{i}$, then $x$ is complete to $B_{2}^{i+1}$, and if $x$ has a non-neighbour in $B_{2}^{i}$, then $x$ is anti-complete to $B_{2}^{i-1}$. If $x \in R_{2}$ has a non-neighbour in $B_{1}^{i}$, then $x$ is anti-complete to $B_{1}^{i+1}$, and if $x$ has a neighbour in $B_{1}^{i}$, then $x$ is complete to $B_{1}^{i-1}$.

Suppose, for contradiction, that $x \in R_{1}$ is adjacent to $y \in B_{2}^{i}$ and non-adjacent to $y^{\prime} \in B_{2}^{i+1}$. Consider a vertex $z \in V_{3}$ that distinguishes $B^{i}$ and $B^{i+1}$. Since $B^{i}<_{B} B^{i+1}$, Claim 2 implies that $z$ is complete to $B_{1}^{i+1} \cup B_{2}^{i}$ and anti-complete to $B_{2}^{i+1} \cup B_{1}^{i}$. 
Now if $x$ is adjacent to $z$ then $G[x, y, z]$ is a rainbow $K_{3}$ and if $x$ is non-adjacent to $z$ then $G\left[x, y^{\prime}, z\right]$ is a rainbow $3 P_{1}$. It follows that if $x \in R_{1}$ has a neighbour in $B_{2}^{i}$ then $x$ is complete to $B_{2}^{i+1}$ and if $x$ has a non-neighbour in $B_{2}^{i+1}$, then $x$ is anti-complete to $B_{2}^{i}$. The claim follows by symmetry.

Claim 5 allows us to update the sequence of blocks as follows:

Update Procedure. For $i \in\{1,2\}$, if $R_{i}$ contains a vertex $x$ that has both a neighbour $y$ and a non-neighbour $y^{\prime}$ in $B_{3-i}^{j}$ for some $j$, we add $x$ to the sets $B_{i}^{j}$ and $B^{j}$ and remove it from $R_{i}$.

Claim 6. Applying the Update Procedure preserves the chain property (see Claim 4) of the blocks $B^{i}$.

Assume that the chain property holds (possibly after some applications of the Update Procedure). Without loss of generality, assume $x \in R_{1}$ has both a neighbour $y$ and a non-neighbour $y^{\prime}$ in some set $B_{2}^{j}$ (the case where $x \in R_{2}$ follows similarly). We will show that the chain property continues to hold after adding $x$ to $B_{1}^{j}$ and $B_{j}$ and removing it from $R_{1}$. Recall that every vertex of $B_{1}^{j}$ has the same neighbourhood in $V_{3}$ by definition of $B^{j}$. We first show that $x$ has the same neighbourhood in $V_{3}$ as the vertices of $B_{1}^{j}$. If $z \in V_{3}$ is non-adjacent to $x$, but complete to $B_{1}^{j}$, then $z$ is anti-complete to $B_{2}^{j}$, so $G\left[x, y^{\prime}, z\right]$ is a rainbow $3 P_{1}$, a contradiction. Similarly, if $z \in V_{3}$ is adjacent to $x$, but anti-complete to $B_{1}^{j}$, then $z$ is complete to $B_{2}^{j}$, so $G[x, y, z]$ is a rainbow $K_{3}$, a contradiction. Therefore $x$ must have the same neighbourhood in $V_{3}$ as the vertices of $B_{1}^{j}$. By Claim 4 , this means that Property (ii) of the chain property is preserved if we apply the Update Procedure with the vertex $x$.

Now suppose that $y^{\prime \prime} \in B_{2}^{k}$ for some $k \neq j$. Then there must be a vertex $z \in V_{3}$ that distinguishes $B^{j}$ and $B^{k}$. If $k<j$ then by Claim 2 the vertex $z$ is complete to $B_{2}^{k} \cup B_{1}^{j}$ and anti-complete to $B_{1}^{k} \cup B_{2}^{j}$, so $x$ must be non-adjacent to $y^{\prime \prime}$, otherwise $G\left[x, y^{\prime \prime}, z\right]$ would be a rainbow $K_{3}$. If $k>j$ then by Claim 2 the vertex $z$ is anti-complete to $B_{2}^{k} \cup B_{1}^{j}$ and complete to $B_{1}^{k} \cup B_{2}^{j}$, so $x$ must be adjacent to $y^{\prime \prime}$, otherwise $G\left[x, y^{\prime \prime}, z\right]$ would be a rainbow $3 P_{1}$. We conclude that Property (i) of the chain property is also preserved if we apply the Update Procedure with the vertex $x$. By symmetry and induction this completes the proof of Claim 6.

By Claim 6 we may therefore apply the Update Procedure exhaustively, after which the chain property will continue to hold. Once this procedure is complete, every remaining vertex of $R_{1}$ will be either complete or anti-complete to each set $B_{2}^{j}$. In fact, by Claim 5, we know that for every vertex $x \in R_{1}$, there is an $i \in\{0, \ldots, p\}$ such that $x$ has a neighbour in all $B_{2}^{j}$ with $j>i$ (if such a $j$ exists) and $x$ has a non-neighbour in all $B_{2}^{j}$ with $j \leq i$ (if any such $j$ exists). Since $x$ is complete or anti-complete to each set $B_{2}^{j}$, we obtain the following conclusion:

- for every vertex $x \in R_{1}$, there is an $i \in\{0, \ldots, p\}$ such that $x$ is complete to all $B_{2}^{j}$ with $j>i$ (if such a $j$ exists) and $x$ is anti-complete to all $B_{2}^{j}$ with $j \leq i$ (if any such $j$ exists). We denote the corresponding subset of $R_{1}$ by $Y_{1}^{i}$.

By symmetry, we also obtain the following:

- for every vertex $x \in R_{2}$, there is an $i \in\{0, \ldots, p\}$ such that $x$ is complete to all $B_{1}^{j}$ with $j \leq i$ (if such a $j$ exists) and $x$ is anti-complete to all $B_{1}^{j}$ with $j>i$ (if any such $j$ exists). We denote the corresponding subset of $R_{2}$ by $Y_{2}^{i}$.

We also partition the vertices of $V_{3}$ into $p+1$ subsets $V_{3}^{0}, \ldots, V_{3}^{p}$ such that the vertices of $V_{3}^{j}$ are complete to $B_{2}^{i}$ and anti-complete to $B_{1}^{i}$ for $i \leq j$ and complete to $B_{1}^{i}$ and anti-complete to $B_{2}^{i}$ for $i>j$. (So $V_{3}^{0}$ is complete to $B_{i}^{1}$ for all $i$ and $V_{3}^{p}$ is complete to $B_{i}^{2}$ for all $i$.)

Claim 7. For each $i$, if $j<i$ then $V_{3}^{i}$ is anti-complete to $Y_{1}^{j}$ and complete to $Y_{2}^{j}$, and if $j>i$ then $V_{3}^{i}$ is complete to $Y_{1}^{j}$ and anti-complete to $Y_{2}^{j}$.

Suppose that $z \in V_{3}^{i}$ and $x \in Y_{1}^{j}$ and $y \in Y_{2}^{j}$ (note that such vertices $x$ and $y$ do not exist if $Y_{1}^{j}$ or $Y_{2}^{j}$, respectively, is empty). First suppose that $j<i$ and choose arbitrary vertices $x^{\prime} \in B_{1}^{i}, y^{\prime} \in B_{2}^{i}$. Note that $x$ and $z$ are both complete to $B_{2}^{i}$ and $y$ and $z$ are both anti-complete to $B_{1}^{i}$. Then $z$ cannot be adjacent to $x$ otherwise $G\left[x, y^{\prime}, z\right]$ would be a rainbow $K_{3}$ and $z$ must be adjacent to $y$, otherwise $G\left[x^{\prime}, y, z\right]$ would be a rainbow $3 P_{1}$. Now suppose $i<j$ and choose arbitrary vertices $x^{\prime} \in B_{1}^{i+1}, y^{\prime} \in B_{2}^{i+1}$. Note that $x$ and $z$ are both anti-complete to $B_{2}^{i+1}$ and $y$ and $z$ are both complete to $B_{1}^{i+1}$. Then $z$ must be adjacent to $x$ otherwise $G\left[x, y^{\prime}, z\right]$ would be a rainbow $3 P_{1}$ and $z$ must be non-adjacent to $y$, otherwise $G\left[x^{\prime}, y, z\right]$ would be a rainbow $K_{3}$. This completes the proof of Claim 7 . 
Let $G^{i}$ denote the subgraph of $G$ induced by $Y_{1}^{i} \cap Y_{2}^{i} \cap V_{3}^{i}$. By Claims 4, 6 and 7 the graph $G$ can be partitioned into slices: $G^{0}, G\left[B^{1}\right], G^{1}, G\left[B^{2}\right], \ldots, G\left[B^{p}\right], G^{p}$. Recall that the graph $G$ is of type $t$ and $G\left[V_{1} \cup V_{2}\right]$ contains an induced $2 P_{2}$. Since $G\left[Y_{1}^{i} \cup Y_{2}^{i}\right]$ is $2 P_{2}$-free (by construction, since the original sequence $H^{1}, H^{2}, \ldots, H^{q}$ of $2 P_{2} s$ was maximal), it follows that each $G^{i}$ is of type at most $t-1$. Furthermore, since each $G\left[B_{i}\right]$ is bipartite, it forms a curious graph in which the set $V_{3}$ is empty, so it has type at most 1 . This completes the proof.

\subsubsection{Curious graphs, clique-width and well-quasi-orderability}

We are now ready to state the main result of this section.

Theorem 4. Let $X$ be a hereditary class of graphs. If the set of bipartite graphs in $X$ is well-quasi-ordered by the labelled induced subgraph relation or has bounded clique-width, then the same property also holds for the set of curious graphs in X.

Proof. Let $X^{\prime}$ be the set of bipartite graphs in $X$ and let $X^{\prime \prime}$ be the set of curious graphs in $X$; note that $X^{\prime}$ is a subset of $X^{\prime \prime}$. By Lemma 13, if $G \in X^{\prime \prime}$ is a curious graph of type 0 or 1 with respect to a partition $\left(V_{1}, V_{2}, V_{3}\right)$, then $G$ can be partitioned into slices from $X^{\prime}$. By Lemma 14 , if $G \in X^{\prime \prime}$ is a curious graph of type 2 with respect to a partition $\left(V_{1}, V_{2}, V_{3}\right)$, then $G$ can be partitioned into slices from $X^{\prime \prime}$ of type at most 1 . By Lemma 14, if $G \in X^{\prime \prime}$ is a curious graph of type 3 with respect to a partition $\left(V_{1}, V_{2}, V_{3}\right)$, then $G$ can be partitioned into slices from $X^{\prime \prime}$ of type at most 2 .

Lemma 11 implies that if a subclass $Z$ of $X^{\prime \prime}$ is well-quasi-ordered by the labelled induced subgraph relation, then the set $Y$ of graphs in $X^{\prime \prime}$ that can be partitioned into slices from $Z$ is also well-quasi-ordered by the labelled induced subgraph relation. We combine this statement with the three statements in the paragraph above. Suppose that $X^{\prime}$ is well-quasiordered by the labelled induced subgraph relation. Then the set of curious graphs in $X^{\prime \prime}$ of type 0 or 1 is well-quasi-ordered by the labelled induced subgraph relation. Thus the set of curious graphs in $X^{\prime \prime}$ of type 2 is also well-quasi-ordered by the labelled induced subgraph relation. Thus the set of curious graphs in $X^{\prime \prime}$ of type 3 is also well-quasi-ordered by the labelled induced subgraph relation. Therefore, if the set of bipartite graphs in $X$ is well-quasi-ordered by the labelled induced subgraph relation, then the set of curious graphs in $X$ is also well-quasi-ordered by the labelled induced subgraph relation.

Lemma 10 implies that if a graph $G \in X^{\prime \prime}$ can be partitioned into slices of clique-width at most $k$, then $G$ has clique-width at most $\max (3 k, 6)$. We combine this statement with the three statements in the first paragraph. Suppose that the graphs in $X^{\prime}$ have bounded clique-width. Then the curious graphs in $X^{\prime \prime}$ of type 0 or 1 also have bounded clique-width. Thus the curious graphs in $X^{\prime \prime}$ of type 2 also have bounded clique-width. Thus the curious graphs in $X^{\prime \prime}$ of type 3 also have bounded clique-width. Therefore, if the set of bipartite graphs in $X$ has bounded clique-width then the set of curious graphs in $X$ also has bounded clique-width. This completes the proof.

\section{Applications of our method}

In this section we show that the class of $\left(K_{3}, P_{1}+P_{5}\right)$-free graphs and the class of $\left(K_{3}, P_{2}+P_{4}\right)$-free graphs have bounded clique-width and are well-quasi-ordered by the labelled induced subgraph relation. To do this, we first prove the two structural lemmas mentioned in Section 3. Recall that these lemmas consider the case where a graph in one of these classes contains an induced subgraph isomorphic to $C_{5}$ and that the main approach used in the proof is to partition the remaining vertices of the graph into sets according to their neighbourhood in this $C_{5}$ and then analyse the edges between the vertices in these sets. As discussed, this enables us to use a bounded number of vertex deletions and bipartite complementations, to transform the graph into a disjoint union of curious graphs and 3-uniform graphs in the respective graph class. We then combine these lemmas with Theorem 3 and Lemma 6 to prove Theorem 5, which is our main result.

The first of the two lemmas is implicit in the proofs of [12, Lemma 9 and Theorem 3], but without an explicit upper bound on the number of operations used and the number of obtained curious graphs. For completeness, we give a direct proof and provide such explicit bounds. Note that the bounds on the number of vertex deletions, bipartite complementations and curious graphs in both lemmas are not tight, but any upper bound on these numbers will be sufficient for our purposes.

Lemma 15. Given any connected $\left(K_{3}, P_{1}+P_{5}\right)$-free graph $G$ that contains an induced $C_{5}$, we can apply at most 5 vertex deletions and at most 31 bipartite complementation operations to obtain a graph $H$ that is the disjoint union of $11\left(K_{3}, P_{1}+P_{5}\right)$-free curious graphs.

Proof. Let $G$ be a connected $\left(K_{3}, P_{1}+P_{5}\right)$-free graph that contains an induced cycle $C$ on the vertices $v_{1}, \ldots, v_{5}$, listed in order along the cycle. To aid notation, for the remainder of the proof subscripts on vertices and on sets should be interpreted modulo 5. We will show how to use vertex deletions and bipartite complementations to partition the graph into a disjoint union of curious graphs. At the end of the proof, we will verify the number of operations used.

Since $G$ is $K_{3}$-free, every vertex not on the cycle $C$ has at most two neighbours on $C$ and if it does have two neighbours on $C$, then these neighbours must be non-consecutive vertices of the cycle. We may therefore partition the vertices in $V(G) \backslash V(C)$ into eleven sets as follows:

- $U$ is the set of vertices anti-complete to $C$, 
- for $i \in\{1, \ldots, 5\}, W_{i}$ is the set of vertices whose unique neighbour on $C$ is $v_{i}$ and

- for $i \in\{1, \ldots, 5\}, V_{i}$ is the set of vertices adjacent to $v_{i-1}$ and $v_{i+1}$ and non-adjacent to the rest of $C$.

We now prove a series of claims. We start by showing how to use a bipartite complementation to disconnect a curious graph containing $U$ from the rest of the graph. This will enable us to assume that $U=\emptyset$ for the remainder of the proof (Claim 1). We then analyse the edges between the remaining non-empty sets (i.e. those of the form $W_{i}$ and $V_{i}$ ) and show that we can use bipartite complementations to disconnect curious graphs containing (subsets of) these sets from the rest of the graph. This will enable us to assume that $V_{i}=W_{i}=\emptyset$ for all $i \in\{1, \ldots, 5\}$ (Claims 10 and 11). After this, the only case left to consider will be when $G=C$, in which case we simply apply five vertex deletions. At the end of the proof, we will count the number of vertex deletions and bipartite complementations used and the number of curious graphs obtained throughout the proof and verify that the bounds specified in the lemma are satisfied.

Claim 1. We may assume that $U=\emptyset$.

Suppose, for contradiction, that there are two vertices $u, u^{\prime} \in U$ that do not have the same neighbourhood in some set $V_{i}$ or $W_{i}$. Say $v \in V_{i} \cup W_{i+1}$ is adjacent to $u$, but not $u^{\prime}$ for some $i \in\{1, \ldots, 5\}$. Note that $v$ is adjacent to $v_{i+1}$, but non-adjacent to $v_{i}, v_{i+2}$ and $v_{i+3}$. Thus $G\left[v_{i+3}, u^{\prime}, u, v, v_{i+1}, v_{i}\right]$ is a $P_{1}+P_{5}$ if $u$ and $u^{\prime}$ are adjacent and $G\left[u^{\prime}, u, v, v_{i+1}, v_{i+2}, v_{i+3}\right]$ is a $P_{1}+P_{5}$ if they are not. This contradiction means that every vertex in $U$ has the same neighbourhood in every set $V_{i}$ and every set $W_{i}$. Since $G$ is connected, there must be a vertex $v$ in some $V_{i}$ or $W_{i}$ that is adjacent to every vertex of $U$. Since $G$ is $K_{3}$-free, $U$ must therefore be an independent set. Applying a bipartite complementation between $U$ and the vertices adjacent to the vertices of $U$ disconnects $G[U]$ from the rest of the graph. Since $U$ is independent, $G[U]$ is a curious graph (with two of the three partition classes empty). This completes the proof of Claim 1.

Claim 2. For $i \in\{1, \ldots, 5\}, V_{i-1} \cup W_{i} \cup V_{i+1}$ is an independent set.

Indeed, if $x, y \in V_{i-1} \cup W_{i} \cup V_{i+1}$ are adjacent then $G\left[x, y, v_{i}\right]$ is a $K_{3}$, a contradiction. This completes the proof of Claim 2.

Claim 3. For $i \in\{1, \ldots, 5\}, W_{i}$ is complete to $W_{i-1} \cup W_{i+1}$.

Indeed, if $x \in W_{i-1}$ is non-adjacent to $y \in W_{i}$ then $G\left[x, y, v_{i}, v_{i+1}, v_{i+2}, v_{i+3}\right]$ is a $P_{1}+P_{5}$, a contradiction. Claim 3 follows by symmetry.

Claim 4. For $i \in\{1, \ldots, 5\}, W_{i}$ is complete or anti-complete to $W_{i+2}$.

Suppose, for contradiction, that $x \in W_{i+2}$ has a neighbour $y \in W_{i}$ and a non-neighbour $y^{\prime} \in W_{i}$. By Claim 2, $y$ is non-adjacent to $y^{\prime}$. Thus $G\left[y^{\prime}, y, x, v_{i+2}, v_{i+3}, v_{i+4}\right]$ is a $P_{1}+P_{5}$, a contradiction. Claim 4 follows by symmetry.

Claim 5. For $i \in\{1, \ldots, 5\}, V_{i}$ is either complete or anti-complete to $W_{i}$.

Suppose, for contradiction, that $x \in V_{i}$ has a neighbour $y \in W_{i}$ and a non-neighbour $y^{\prime} \in W_{i}$. By Claim 2, $y$ is nonadjacent to $y^{\prime}$. Thus $G\left[y^{\prime}, y, x, v_{i+1}, v_{i+2}, v_{i+3}\right]$ is a $P_{1}+P_{5}$, a contradiction. Therefore every vertex in $V_{i}$ is complete or anti-complete to $W_{i}$. Now suppose, for contradiction, that $y \in W_{i}$ has a neighbour $x \in V_{i}$ and a non-neighbour $x^{\prime} \in V_{i}$. By Claim 2, $x$ is non-adjacent to $x^{\prime}$. Thus $G\left[v_{i+3}, v_{i}, y, x, v_{i+1}, x^{\prime}\right]$ is a $P_{1}+P_{5}$, a contradiction. Therefore every vertex in $W_{i}$ is complete or anti-complete to $V_{i}$. This completes the proof of Claim 5.

Claim 6. For $i \in\{1, \ldots, 5\}$, every vertex of $V_{i}$ is complete to either $V_{i-1}$ or $V_{i+1}$.

Suppose, for contradiction, that $x \in V_{i}$ is non-adjacent to $y \in V_{i-1}$ and $z \in V_{i+1}$. By Claim 2, $y$ is non-adjacent to $z$. Thus $G\left[y, v_{i-1}, x, v_{i+1}, v_{i+2}, z\right]$ is a $P_{1}+P_{5}$, a contradiction. This completes the proof of Claim 6.

By Claim 6, we can partition each set $V_{i}$ into three (possibly empty) subsets $V_{i}^{0}, V_{i}^{-}$and $V_{i}^{+}$as follows:

- $V_{i}^{0}$ is the set of vertices in $V_{i}$ that dominate both $V_{i-1}$ and $V_{i+1}$.

- $V_{i}^{-}$is the set of vertices in $V_{i} \backslash V_{i}^{0}$ that dominate $V_{i-1}$ (and so have non-neighbours in $V_{i+1}$ ).

- $V_{i}^{+}$is the set of vertices in $V_{i} \backslash V_{i}^{0}$ that dominate $V_{i+1}$ (and so have non-neighbours in $V_{i-1}$ ).

Note that by doing this we partition the vertices of $V_{1} \cup \ldots \cup V_{5}$ into a total of 15 subsets (some or all of which may be empty). By definition of this partition, if $x \in V_{i}$ is non-adjacent to $y \in V_{i+1}$, then $x \in V_{i}^{-}$and $y \in V_{i+1}^{+}$. Moreover, every 
vertex of $V_{i}^{-}$has a non-neighbour in $V_{i+1}^{+}$and vice versa. Note that for $i \in\{1, \ldots, 5\}$ the sets $V_{i}^{0}, V_{i}^{-}, V_{i}^{+}, V_{i+2}^{0}, V_{i+2}^{-}$ and $V_{i+2}^{+}$are pairwise anti-complete by Claim 2. Furthermore, for $i \in\{1, \ldots, 5\}, V_{i}^{0}$ and $V_{i}^{+}$are complete to $V_{i+1}^{0}, V_{i+1}^{-}$ and $V_{i+1}^{+}$, and $V_{i}^{-}$is complete to $V_{i+1}^{0}$ and $V_{i+1}^{-}$. Therefore, for nearly every pair $\{X, Y\}$ of these 15 subsets, $X$ and $Y$ are either complete or anti-complete to each other. The only possible exceptions are the five disjoint pairs of the form $\left\{V_{i}^{-}, V_{i+1}^{+}\right\}$.

Next, for each $i \in\{1, \ldots, 5\}$ we analyse the edges between $W_{i}$ and the subsets of $V_{i-2}$ and $V_{i+2}$.

Claim 7. For $i \in\{1, \ldots, 5\}, W_{i}$ is anti-complete to $V_{i-2}^{-} \cup V_{i+2}^{+}$.

Suppose, for contradiction, that $x \in W_{i}$ has a neighbour $y \in V_{i+2}^{+}$. By definition of $V_{i+2}^{+}, y$ must have a non-neighbour $z \in V_{i+1}$. By Claim 2, $z$ is non-adjacent to $x$. Thus $G\left[v_{i-1}, x, y, v_{i+1}, v_{i+2}, z\right]$ is a $P_{1}+P_{5}$, a contradiction. Claim 7 follows by symmetry.

Claim 8. For $i \in\{1, \ldots, 5\}$, if $x \in W_{i}, y \in V_{i+2}$ and $z \in V_{i-2}$ then $G[x, y, z]$ is not a $3 P_{1}$.

Indeed, if $x \in W_{i}, y \in V_{i+2}$ and $z \in V_{i-2}$ and $G[x, y, z]$ is a $3 P_{1}$ then $G\left[z, x, v_{i}, v_{i+1}, y, v_{i-2}\right]$ is a $P_{1}+P_{5}$, a contradiction. This completes the proof of Claim 8.

Claim 9. For $i \in\{1, \ldots, 5\}$ :

(i) If $x \in W_{i}$ has a neighbour in $V_{i+2}^{0}$, then $x$ is anti-complete to $V_{i+3}$ and complete to $V_{i+2}^{-}$.

(ii) If $x \in W_{i}$ has a neighbour in $V_{i+3}^{0}$, then $x$ is anti-complete to $V_{i+2}$ and complete to $V_{i+3}^{+}$.

Suppose $x \in W_{i}$ has a neighbour $y \in V_{i+2}^{0}$. If $z \in V_{i+3}$ then $y$ is adjacent to $z$, so $x$ must be non-adjacent to $z$, otherwise $G[x, y, z]$ would be a $K_{3}$. Therefore $x$ is anti-complete to $V_{i+3}$. If $y^{\prime} \in V_{i+2}^{-}$then $y^{\prime}$ has a non-neighbour $z^{\prime} \in V_{i+3}$. Note that $z^{\prime}$ is non-adjacent to $x$. Now $x$ must be adjacent to $y^{\prime}$, otherwise $G\left[x, y^{\prime}, z^{\prime}\right]$ would be a $3 P_{1}$, contradicting Claim 8. It follows that $x$ is complete to $V_{i+2}^{-}$. Claim 9 follows by symmetry.

Recall that $W_{i}$ is anti-complete to $V_{i-2}^{-} \cup V_{i+2}^{+}$by Claim 7. By Claim 9 we can partition $W_{i}$ into three (possibly empty) subsets as follows:

- $W_{i}^{2}$ the set of vertices in $W_{i}$ that have neighbours in $V_{i+2}^{0}$ (and are therefore anti-complete to $V_{i+3}$ and complete to $\left.V_{i+2}^{-}\right)$,

- $W_{i}^{3}$ the set of vertices in $W_{i}$ that have neighbours in $V_{i+3}^{0}$ (and are therefore anti-complete to $V_{i+2}$ and complete to $\left.V_{i+3}^{+}\right)$and

- $W_{i}^{0}$ the set of vertices in $W_{i} \backslash\left(W_{i}^{2} \cup W_{i}^{3}\right)$ (which are therefore anti-complete to $V_{i+2}^{0} \cup V_{i+3}^{0}$ ).

Claim 10. For $i \in\{1, \ldots, 5\}$, we may assume that $W_{i}^{0} \cup V_{i-2}^{+} \cup V_{i+2}^{-}=\emptyset$.

We first show how to remove all edges from vertices in $G\left[W_{i}^{0} \cup V_{i-2}^{+} \cup V_{i+2}^{-}\right]$to the rest of $G$ by using three bipartite complementations. In other words, we will use three bipartite complementations to remove all edges between $G\left[W_{i}^{0} \cup\right.$ $\left.V_{i-2}^{+} \cup V_{i+2}^{-}\right]$and $G\left[C \cup V_{i-1} \cup V_{i} \cup V_{i+1} \cup V_{i+2}^{0} \cup V_{i+2}^{+} \cup V_{i+3}^{0} \cup V_{i+3}^{-} \cup W_{i-1} \cup W_{i}^{2} \cup W_{i}^{3} \cup W_{i+1} \cup W_{i+2} \cup W_{i+3}\right]$.

By Claim $2, W_{i}^{0}$ is anti-complete to $W_{i}^{2}, W_{i}^{3}, V_{i-1}$ and $V_{i+1}$. By Claim $7, W_{i}^{0}$ is anti-complete to $V_{i+3}^{-}$and $V_{i+2}^{+}$. By definition, $W_{i}^{0}$ is anti-complete to $V_{i+2}^{0}$ and $V_{i+3}^{0}$. By Claim $3, W_{i}^{0}$ is complete to $W_{i-1}$ and $W_{i+1}$. By Claim $5, W_{i}^{0}$ is either complete or anti-complete to $V_{i}$. By Claim $4, W_{i}^{0}$ is either complete or anti-complete to $W_{i+2}$ and either complete or anti-complete to $W_{i+3}$. By definition of $W_{i}, v_{i}$ is complete to $W_{i}$ and $C \backslash\left\{v_{i}\right\}$ is anti-complete to $W_{i}$. Therefore, by applying at most one bipartite complementation, we can remove all edges with one end-vertex in $W_{i}^{0}$ and the other end-vertex outside $W_{i}^{0} \cup V_{i-2}^{+} \cup V_{i+2}^{-}$.

By Claim 2, $V_{i+2}^{-}$is anti-complete to $V_{i}, V_{i-1}, V_{i+2}^{+}, V_{i+2}^{0}, W_{i+1}$ and $W_{i+3}$. By Claim $5, V_{i+2}^{-}$is either complete or anti-complete to $W_{i+2}$. By Claim $7, V_{i+2}^{-}$is anti-complete to $W_{i-1}$. By definition, $V_{i+2}^{-}$is complete to $V_{i+1}$. By definition of $V_{i+3}^{0}$ and $V_{i+3}^{-}, V_{i+2}^{-}$is complete to $V_{i+3}^{0}$ and $V_{i+3}^{-}$. By Claim $9, V_{i+2}^{-}$is complete to $W_{i}^{2}$ and anti-complete to $W_{i}^{3}$. By definition of $V_{i+2},\left\{v_{i+1}, v_{i+3}\right\}$ is complete to $V_{i+2}^{-}$and $C \backslash\left\{v_{i+1}, v_{i+3}\right\}$ is anti-complete to $V_{i+2}^{-}$. Therefore, by applying at most one bipartite complementation, we can remove all edges with one end-vertex in $V_{i+2}^{-}$and the other end-vertex outside $W_{i}^{0} \cup V_{i-2}^{+} \cup V_{i+2}^{-}$. Symmetrically, by applying at most one bipartite complementation, we can remove all edges with one end-vertex in $V_{i-2}^{+}$and the other end-vertex outside $W_{i}^{0} \cup V_{i-2}^{+} \cup V_{i+2}^{-}$. 
Thus we can apply three bipartite complementations to disconnect $G\left[W_{i}^{0} \cup V_{i-2}^{+} \cup V_{i+2}^{-}\right]$from the rest of the graph. By Claim 2, $W_{i}^{0}, V_{i-2}^{+}$and $V_{i+2}^{-}$are independent sets. Therefore, by Claim 8, $G\left[W_{i}^{0} \cup V_{i-2}^{+} \cup V_{i+2}^{-}\right]$is a curious graph. This completes the proof of Claim 10.

By the Claim 10, the only sets that remain non-empty are ones of form $W_{i}^{3}, W_{i}^{2}$ or $V_{i}^{0}$. We prove the following claim.

Claim 11. For $i \in\{1, \ldots, 5\}$, we may assume that $W_{i}^{3} \cup W_{i+1}^{2} \cup V_{i-2}^{0}=\emptyset$.

We first show how to remove all edges from vertices in $G\left[W_{i}^{3} \cup W_{i+1}^{2} \cup V_{i-2}^{0}\right]$ to the rest of the graph by using three bipartite complementations. In other words, we will use three bipartite complementations to remove all edges between $G\left[W_{i}^{3} \cup W_{i+1}^{2} \cup V_{i-2}^{0}\right]$ and $G\left[C \cup V_{i-1}^{0} \cup V_{i}^{0} \cup V_{i+1}^{0} \cup V_{i+2}^{0} \cup W_{i-2} \cup W_{i-1} \cup W_{i}^{2} \cup W_{i+1}^{3} \cup W_{i+2}\right]$.

By Claim $2, W_{i}^{3}$ is anti-complete to $W_{i}^{2}, V_{i-1}^{0}$ and $V_{i+1}^{0}$. By Claim $3, W_{i}^{3}$ is complete to $W_{i-1}$ and $W_{i+1}^{3}$. By Claim 4 , $W_{i}^{3}$ is either complete or anti-complete to $W_{i+2}$ and either complete or anti-complete to $W_{i-2}$. By Claim $5, W_{i}^{3}$ is either complete or anti-complete to $V_{i}^{0}$. By Claim $9, W_{i}^{3}$ is anti-complete to $V_{i+2}^{0}$. By definition of $W_{i}, v_{i}$ is complete to $W_{i}^{3}$ and $C \backslash\left\{v_{i}\right\}$ is anti-complete to $W_{i}^{3}$. Therefore, by applying at most one bipartite complementation, we can remove all edges with one end-vertex in $W_{i}^{3}$ and the other end-vertex outside $W_{i}^{3} \cup W_{i+1}^{2} \cup V_{i-2}^{0}$. Symmetrically, by applying at most one bipartite complementation, we can remove all edges with one end-vertex in $W_{i+1}^{2}$ and the other end-vertex outside $W_{i}^{3} \cup W_{i+1}^{2} \cup V_{i-2}^{0}$.

By Claim 2, $V_{i-2}^{0}$ anti-complete to $V_{i}, W_{i-1}, W_{i+2}, V_{i+1}$. By Claim 5, $V_{i-2}^{0}$ is either complete or anti-complete to $W_{i-2}$. By definition, $V_{i-2}^{0}$ is complete to $V_{i-1}^{0}$ and $V_{i-3}^{0}$. By Claim 9, $V_{i-2}^{0}$ is anti-complete to $W_{i}^{2}$ and $W_{i+1}^{3}$. By definition of $V_{i-2}$, $\left\{v_{i-1}, v_{i-3}\right\}$ is complete to $V_{i-2}^{0}$ and $C \backslash\left\{v_{i-1}, v_{i-3}\right\}$ is anti-complete to $V_{i-2}^{0}$. Therefore, by applying at most one bipartite complementation, we can remove all edges with one end-vertex in $V_{i-2}^{0}$ and the other end-vertex outside $W_{i}^{3} \cup W_{i+1}^{2} \cup V_{i-2}^{0}$.

Thus we can apply three bipartite complementations to disconnect $G\left[W_{i}^{3} \cup W_{i+1}^{2} \cup V_{i-2}^{0}\right]$ from the rest of the graph. By Claim $2, W_{i}^{3}, W_{i+1}^{2}$ and $V_{i-2}^{0}$ are independent sets. By Claim 3, $W_{i}^{3}$ is complete to $W_{i+1}^{2}$, so $G\left[W_{i}^{3} \cup W_{i+1}^{2} \cup V_{i-2}^{0}\right]$ is a curious graph. This completes the proof of Claim 11.

By Claim 11, we may assume that $G$ only contains the five vertices in the cycle $C$. We delete these vertices.

To complete the proof, it remains to verify the number of operations applied and the number of obtained curious graphs. In Claim 1, we apply one bipartite complementation and obtain one curious graph. In Claim 10 , for each $i \in\{1, \ldots, 5\}$, we apply three bipartite complementations and obtain one curious graph. In Claim 11, for each $i \in\{1, \ldots, 5\}$, we apply three bipartite complementations and obtain one curious graph. Finally, we apply five vertex deletions to delete the cycle $C$ from the graph. This leads to a total of five vertex deletions, $1+(5 \times 3)+(5 \times 3)=31$ bipartite complementations and $1+5+5=11$ obtained curious graphs.

We now prove our second structural lemma.

Lemma 16. Given any prime $\left(K_{3}, P_{2}+P_{4}\right)$-free graph $G$ that contains an induced $C_{5}$, we can apply at most 2570 vertex deletions and at most 459 bipartite complementation operations to obtain a graph $\mathrm{H}$ that is the disjoint union of at most $19\left(K_{3}, P_{2}+P_{4}\right)$-free curious graphs and at most one 3-uniform graph.

Proof. Let $G$ be a prime $\left(K_{3}, P_{2}+P_{4}\right)$-free graph that contains an induced cycle $C$ on the vertices $v_{1}, \ldots, v_{5}$, listed in order along the cycle. To aid notation, for the remainder of the proof subscripts on vertices and on sets should be interpreted modulo 5. We will show how to use vertex deletions and bipartite complementations to partition the graph into a disjoint union of curious graphs and 3-uniform graphs. At the end of the proof, we will verify the number of operations used.

Since $G$ is $K_{3}$-free, every vertex not on the cycle $C$ has at most two neighbours on $C$ and if it does have two neighbours on $C$, then these neighbours must be non-consecutive vertices of the cycle. We may therefore partition the vertices in $V(G) \backslash V(C)$ into eleven sets as follows:

- $U$ is the set of vertices anti-complete to $C$,

- for $i \in\{1, \ldots, 5\}, W_{i}$ is the set of vertices whose unique neighbour on $C$ is $v_{i}$ and

- for $i \in\{1, \ldots, 5\}, V_{i}$ is the set of vertices adjacent to $v_{i-1}$ and $v_{i+1}$ and non-adjacent to the rest of $C$.

We now prove a series of claims. We start by showing how to use a bounded number of vertex deletions and bipartite complementations to disconnect a 3-uniform or bipartite induced subgraph containing $U$ from the rest of the graph. This will enable us to assume (Claim 25) that $U=\emptyset$ for the remainder of the proof. Similarly, we analyse the edges between the vertices in the other sets (of the form $V_{i}$ and $W_{i}$ ) defined above and show that we can use bipartite complementations to disconnect curious graphs containing (subsets of) these sets from the rest of the graph (Claims 25, 27, 30, 31, 32 and 33). 
This will enable us to assume that $W_{i}=\varnothing$ for all $i \in\{1, \ldots, 5\}$ and that all remaining vertices in sets $V_{i}$ for $i \in\{1, \ldots, 5\}$ have very restricted neighbourhoods (that is, we will be able to assume that $V_{i}=V_{i}^{0}$ for every $i \in\{1, \ldots, 5\}$, where $V_{i}^{0}$ is a subset of $V_{i}$ defined later in the proof). Under these assumptions, it will be sufficient to then apply five more bipartite complementations to transform $G$ into a curious graph. At the end of the proof, we will count the number of vertex deletions and bipartite complementations used and the number of 3-uniform and curious graphs obtained throughout the proof and verify that the bounds specified in the lemma are satisfied.

Claim 1. For $i \in\{1, \ldots, 5\}, U \cup W_{i}$ is an independent set.

Indeed, if $x, y \in U \cup W_{i}$ are adjacent then $G\left[x, y, v_{i+1}, v_{i+2}, v_{i+3}, v_{i+4}\right]$ is a $P_{2}+P_{4}$, a contradiction. This completes the proof of Claim 1.

Claim 2. For $i \in\{1, \ldots, 5\}, V_{i-1} \cup W_{i} \cup V_{i+1}$ is an independent set.

Indeed, if $x, y \in V_{i-1} \cup W_{i} \cup V_{i+1}$ are adjacent then $G\left[x, y, v_{i}\right]$ is a $K_{3}$, a contradiction. This completes the proof of Claim 2 .

Claim 3. For $i \in\{1, \ldots, 5\}, G\left[U \cup V_{i}\right]$ is $P_{4}$-free and every component of $G\left[U \cup V_{i}\right]$ is a complete bipartite graph.

Indeed, if $x_{1}, x_{2}, x_{3}, x_{4} \in U \cup V_{i}$ induce a $P_{4}$ then $G\left[v_{i+2}, v_{i+3}, x_{1}, x_{2}, x_{3}, x_{4}\right]$ would be a $P_{2}+P_{4}$, a contradiction. Therefore $G\left[U \cup V_{i}\right]$ is indeed $P_{4}$-free. By Claims 1 and 2, the sets $U$ and $V_{i}$ are independent. Therefore $G\left[U \cup V_{i}\right]$ is $P_{4}$-free bipartite graph, so every component of $G\left[U \cup V_{i}\right]$ is a complete bipartite graph. This completes the proof of Claim 3 .

Claim 4. For $i \in\{1, \ldots, 5\}$, every vertex in $V_{i}$ is either complete or anti-complete to $W_{i}$.

Indeed, suppose for contradiction that $x \in V_{i}$ has a neighbour $y \in W_{i}$ and a non-neighbour $y^{\prime} \in W_{i}$. By Claim $1, y$ is non-adjacent to $y^{\prime}$. It follows that $G\left[v_{i+2}, v_{i+3}, x, y, v_{i}, y^{\prime}\right]$ is a $P_{2}+P_{4}$, a contradiction. This completes the proof of Claim 4 .

For $i \in\{1, \ldots, 5\}$, let $V_{i}^{*}$ denote the set of vertices in $V_{i}$ that have neighbours in $U$ and let $V_{i}^{0}=V_{i} \backslash V_{i}^{*}$. Let $V^{*}=\bigcup V_{i}^{*}$. We will show how to use five bipartite complementations to separate $G\left[U \cup V^{*}\right]$ from the rest of the graph. Note that by definition and by Claim 1 , no vertex of $U$ has a neighbour outside $V^{*}$. It is therefore sufficient to show how to disconnect $V^{*}$ from the vertices outside $U \cup V^{*}$.

In fact, for vertices in $V_{i}^{*}$ we can prove a stronger version of Claim 4.

Claim 5. For $i \in\{1, \ldots, 5\}, V_{i}^{*}$ is anti-complete to $W_{i}$.

Indeed, suppose for contradiction that $x \in V_{i}^{*}$ is adjacent to $y \in W_{i}$ and let $z \in U$ be a neighbour of $x$. By Claim $1, y$ is non-adjacent to $z$. It follows that $G\left[v_{i+2}, v_{i+3}, v_{i}, y, x, z\right]$ is a $P_{2}+P_{4}$, a contradiction. This completes the proof of Claim 5 .

Claim 6. For $i \in\{1, \ldots, 5\}$, if $x \in V_{i}^{*}$ is adjacent to $y \in U$ then every vertex $z \in V_{i-1} \cup V_{i+1}$ has exactly one neighbour in $\{x, y\}$.

Indeed, suppose $x \in V_{i}^{*}, y \in U$ and $z \in V_{i-1}$ with $x$ adjacent to $y$. If $z$ is adjacent to both $x$ and $y$ then $G[x, y, z]$ is a $K_{3}$, a contradiction. If $z$ is non-adjacent to both $x$ and $y$ then $G\left[x, y, v_{i}, z, v_{i+3}, v_{i+2}\right]$ is a $P_{2}+P_{4}$, a contradiction. Claim 6 follows by symmetry.

Claim 7. For $i \in\{1, \ldots, 5\}, V_{i}^{*}$ is complete to $V_{i-1}^{0} \cup V_{i+1}^{0}$.

This follows from Claim 6 by definition of $V_{i}^{*}$ and $V_{i-1}^{0} \cup V_{i+1}^{0}$. This completes the proof of Claim 7 .

Claim 8. For $i \in\{1, \ldots, 5\}, V_{i}^{*}$ is complete to $W_{i-2} \cup W_{i+2}$.

Suppose, for contradiction, that $x \in V_{i}^{*}$ is non-adjacent to a vertex $y \in W_{i+2}$ and let $z \in U$ be a neighbour of $x$. By Claim $1, y$ is non-adjacent to $z$. Therefore $G\left[y, v_{i+2}, v_{i}, v_{i-1}, x, z\right]$ is a $P_{2}+P_{4}$, a contradiction. Claim 8 follows by symmetry. 
Claim 9. For $i \in\{1, \ldots, 5\}, V_{i}^{*}$ is complete to $V_{i-1}^{0} \cup V_{i+1}^{0} \cup W_{i-2} \cup W_{i+2} \cup\left\{v_{i-1}, v_{i+1}\right\}$ and anti-complete to every vertex of $G \backslash\left(V^{*} \cup U\right)$ that is not in $V_{i-1}^{0} \cup V_{i+1}^{0} \cup W_{i-2} \cup W_{i+2} \cup\left\{v_{i-1}, v_{i+1}\right\}$. Furthermore, no vertex in $U$ has a neighbour in $G \backslash\left(V^{*} \cup U\right)$.

By definition of $V_{i}^{*}$, every vertex in $V_{i}^{*}$ is complete to $\left\{v_{i-1}, v_{i+1}\right\}$ and anti-complete to $C \backslash\left\{v_{i-1}, v_{i+1}\right\}$. By Claim 2 , $V_{i}^{*}$ is anti-complete to $W_{i-1} \cup W_{i+1} \cup V_{i-2}^{0} \cup V_{i+2}^{0} \cup V_{i}^{0}$. By Claim 7, $V_{i}^{*}$ is complete to $V_{i-1}^{0} \cup V_{i+1}^{0}$. By Claim 5, $V_{i}^{*}$ is anti-complete to $W_{i}$. By Claim $8, V_{i}^{*}$ is complete to $W_{i-2} \cup W_{i+2}$. By definition of $U$, every vertex in $U$ is anti-complete to $C$. By Claim $1, U$ is anti-complete to $W_{j}$ for all $j \in\{1, \ldots, 5\}$ and by definition of $V_{j}^{0}, U$ is non-adjacent to $V_{j}^{0}$ for all $j \in\{1, \ldots, 5\}$. This completes the proof of Claim 9 .

Recall that at the start of the proof we assumed that the graph $G$ is prime. By Claim 9 if for each $i \in\{1, \ldots, 5\}$ we apply a bipartite complementation between $V_{i}^{*}$ and $V_{i-1}^{0} \cup V_{i+1}^{0} \cup W_{i-2} \cup W_{i+2} \cup\left\{v_{i-1}, v_{i+1}\right\}$ then this would remove all edges between $V^{*} \cup U$ and $V(G) \backslash\left(V^{*} \cup U\right)$ and leave all other edges unchanged. However, after doing this, the result might be a graph that is not prime. As such, we will not use Claim 9 to disconnect $G\left[V^{*} \cup U\right]$ from the rest of the graph at this stage, but wait until later in the proof, when we no longer require the property that $G$ is prime. Next, we analyse the structure of $G\left[V^{*} \cup U\right]$. Note that $V_{1}, \ldots, V_{5}$ and $U$ are independent sets by Claims 2 and 1 . By Claim 3, every component of $G\left[U \cup V_{i}\right]$ is a complete bipartite graph. Fix $i \in\{1, \ldots, 5\}$ and consider a component $C^{\prime}$ of $G\left[U \cup V_{i}^{*}\right]$ containing at least one vertex of $V_{i}^{*}$. Let $T=V\left(C^{\prime}\right) \cap V_{i}^{*}$ and $S=V\left(C^{\prime}\right) \cap U$. Note that since every vertex of $V_{i}^{*}$ contains at least one neighbour in $U$ it follows that $|S| \geq 1$. We prove a series of claims.

Claim 10. $|T|=1$.

We first show that $T$ is a module in $G$. Since $T \subseteq V_{i}^{*}$, if a vertex $v$ in $G$ can distinguish two vertices in $T$ then $v \in V^{*} \cup U$ by Claim 9. Since $U$ and $V_{i}^{*}$ are independent sets by Claims 1 and 2, respectively and every component of $G\left[U \cup V_{i}^{*}\right]$ is a complete bipartite graph by Claim 3, no vertex of $\left(U \cup V_{i}^{*}\right) \backslash T$ can distinguish two vertices in $T$. By Claim 2 , no vertex of $V_{i-2}^{*} \cup V_{i+2}^{*}$ can distinguish two vertices in $T$. Because $G[S \cup T]$ is a complete bipartite graph, Claim 6 implies that every vertex of $V_{i-1}^{*} \cup V_{i+1}^{*}$ is either complete to $S$ and anti-complete to $T$ or anti-complete to $S$ and complete to $T$, so no vertex of $V_{i-1}^{*} \cup V_{i+1}^{*}$ can distinguish two vertices in $T$. It follows that $T$ is a module in $G$. Since $G$ is prime, we conclude that $|T|=1$. This completes the proof of Claim 10 .

Claim 11. If $\left|V_{i}^{*}\right|>1$, then $|S|=1$.

We show that if $\left|V_{i}^{*}\right|>1$ then $S$ is a module in $G$. Suppose, for contradiction, that $S$ is not a module in $G$. Since $S \subseteq U$, if a vertex $v$ in $G$ can distinguish two vertices in $S$ then $v \in V^{*} \cup U$ by Claim 9. Since $U$ and $V_{i}^{*}$ are independent sets by Claims 1 and 2, respectively and every component of $G\left[U \cup V_{i}^{*}\right]$ is a complete bipartite graph by Claim 3, no vertex of $\left(U \cup V_{i}^{*}\right) \backslash S$ can distinguish two vertices in $S$. Because $G[S \cup T]$ is a complete bipartite graph, Claim 6 implies that every vertex of $V_{i-1}^{*} \cup V_{i+1}^{*}$ is either complete to $S$ and anti-complete to $T$ or anti-complete to $S$ and complete to $T$, so no vertex of $V_{i-1}^{*} \cup V_{i+1}^{*}$ can distinguish two vertices in $S$. Therefore, there must be a vertex in $V_{i-2} \cup V_{i+2}$ that distinguishes two vertices of $S$. Without loss of generality, assume that there is a vertex $x \in V_{i+2}$ that is adjacent to $y \in S$ and non-adjacent to $y^{\prime} \in S$. Let $z$ be a vertex of $T$, i.e. a vertex of $V_{i}^{*}$ that is adjacent to both $y$ and $y^{\prime}$. Note that $\left|V_{i}^{*}\right|>1$ and any two vertices of $V_{i}^{*}$ belong to different connected components of $G\left[V_{i}^{*} \cup U\right]$ by Claim 10. This means that there must be a vertex $z^{\prime} \in V_{i}^{*} \backslash\{z\}$ and it must have a neighbour $y^{\prime \prime} \in U \backslash S$. Note that $x$ is non-adjacent to $z$ and $z^{\prime}$ by Claim 2 . It follows that $G\left[y^{\prime}, z, v_{i+2}, v_{i+3}, x, y^{\prime \prime}\right]$ or $G\left[y^{\prime \prime}, z^{\prime}, x, y, z, y^{\prime}\right]$ is a $P_{2}+P_{4}$ if $x$ is adjacent or non-adjacent to $y^{\prime \prime}$, respectively. This contradiction implies that $S$ is indeed a module. Since $G$ is prime, we conclude that $|S|=1$. This completes the proof of Claim 11.

Claim 12. If $\left|V_{i}^{*}\right|>1$, then $\left|V_{i-2}^{*}\right| \leq 1$ and $\left|V_{i+2}^{*}\right| \leq 1$.

Suppose, for contradiction, that $V_{i}^{*}$ and $V_{i+2}^{*}$ each contain at least two vertices. Then by Claims 10 and 11 , every connected component of $G\left[V_{i}^{*} \cup U\right]$ containing a vertex of $V_{i}^{*}$ consists of a single edge and since $V_{i}^{*}$ contains at least two vertices, there must be at least two such components. Similarly, there must be two such components in $G\left[V_{i+2}^{*} \cup U\right]$. Therefore we can find $x \in V_{i}^{*}$ adjacent to $y \in U$ and $y^{\prime} \in U \backslash\{y\}$ adjacent to $z \in V_{i+2}^{*}$. By Claim 2, $x$ is non-adjacent to $z$, so $G\left[x, y, y^{\prime}, z, v_{i+3}, v_{i+2}\right]$ is a $P_{2}+P_{4}$, a contradiction. Claim 12 follows by symmetry.

Claim 13. There is a set $V^{* *} \subseteq V^{*}$ with $\left|V^{* *}\right| \leq 5$ such that $G\left[\left(V^{*} \backslash V^{* *}\right) \cup U\right]$ is either bipartite or 3-uniform.

Let $V^{* *}$ be the union of all sets $V_{i}^{*}$ with $i \in\{1, \ldots, 5\}$ such that $\left|V_{i}^{*}\right|=1$. Then $\left|V^{* *}\right| \leq 5$. Consider the graph $H=$ $G\left[\left(V^{*} \backslash V^{* *}\right) \cup U\right]$. For every $i \in\{1, \ldots, 5\}$ the set $V_{i}^{*}$ either contains zero or at least two vertices in $H$. If at most one set $V_{i}^{*}$ contains two vertices in $H$ then by Claims 1 and 2, $H$ is a bipartite graph and we are done. It remains to consider the case when two sets $V_{i}^{*}$ and $V_{j}^{*}$ contain two vertices in $H$. In this case, by Claim 12, $v_{i}$ and $v_{j}$ must be consecutive 
vertices of the cycle $C$ and all other sets $V_{k}^{*}$ contain no vertices of $H$, so we may assume $j=i+1$ and $H=G\left[V_{i}^{*} \cup V_{i+1}^{*} \cup U\right]$. We will show that this graph is 3-uniform.

By Claims 1 and 2, $V_{i}^{*}, V_{i+1}^{*}$ and $U$ are independent sets. By Claims 10 and 11, every vertex of $V_{i}^{*} \cup V_{i+1}^{*}$ has exactly one neighbour in $U$ and every vertex of $U$ has at most one neighbour in $V_{i}^{*}$ and at most one neighbour in $V_{i+1}^{*}$.

If $x \in V_{i}^{*}$ and $y \in V_{i+1}^{*}$ have a common neighbour $z \in U$ then $x$ must be non-adjacent to $y$ by Claim 6 . If $x \in V_{i}^{*}$ is non-adjacent to $y \in V_{i+1}^{*}$ and $z$ is the neighbour of $x$ in $U$ then $y$ must adjacent to $z$ by Claim 6. Therefore a vertex $x \in V_{i}^{*}$ is non-adjacent to a vertex $y \in V_{i+1}^{*}$ if and only if they have the same unique neighbour $z \in U$.

Now applying a bipartite complementation between $V_{i}^{*}$ and $V_{i+1}^{*}$, we obtain a graph every component of which is an induced subgraph of $K_{3}$, with at most one vertex of each component in each of $V_{i}^{*}, V_{i+1}^{*}$ and $U$. Therefore $H$ is a 3-uniform graph. (In terms of the definition of 3 -uniform graphs, we have $k=3, F_{k}=K_{3}$ and $K$ is the $3 \times 3$ matrix which has $K_{1,2}=K_{2,1}=1$ and is zero everywhere else.) This completes the proof of Claim 13.

Claims 9 and 13 would allow us to remove $G\left[V^{*} \cup U\right]$ from the graph. However, if we did this, then we might violate the property that $G$ is prime. Hence, as discussed earlier, we do not actually do so at this stage in the proof. Informally, we may think of the vertices in $V^{*} \cup U$ as having been "dealt with" and we now concern ourselves with the remainder of the graph i.e. the vertices in $C \cup V_{1}^{0} \cup \ldots \cup V_{5}^{0} \cup W_{1} \cup \cdots \cup W_{5}$. First, we look at the sets $W_{i}$ and the edges between these sets.

Claim 14. For $i \in\{1, \ldots, 5\}, W_{i}$ is either complete or anti-complete to $W_{i+1}$.

Suppose, for contradiction, that $x \in W_{i}$ is adjacent to $y \in W_{i+1}$ and non-adjacent to $y^{\prime} \in W_{i+1}$. By Claim $1, y$ is nonadjacent to $y^{\prime}$. Thus $G\left[v_{i-1}, v_{i-2}, x, y, v_{i+1}, y^{\prime}\right]$ is a $P_{2}+P_{4}$, a contradiction. By symmetry, this completes the proof of Claim 14.

In fact, we can strengthen Claim 14 as follows.

Claim 15. For $i \in\{1, \ldots, 5\}, W_{i}$ is either complete or anti-complete to $W_{i-1} \cup W_{i+1}$.

If $W_{i-1}$ or $W_{i+1}$ is empty then the claim follows by Claim 14. Now suppose, for contradiction, that $x \in W_{i}$ has a neighbour $y \in W_{i-1}$ and a non-neighbour $z \in W_{i+1}$. Then $G\left[v_{i+2}, v_{i+3}, v_{i}, x, y, z\right]$ or $G\left[x, y, z, v_{i+1}, v_{i+2}, v_{i+3}\right]$ is a $P_{2}+P_{4}$ if $y$ is adjacent or non-adjacent to $z$, respectively. This contradiction completes the proof of Claim 15 .

Claim 16. For $i \in\{1, \ldots, 5\}, G\left[W_{i} \cup W_{i+2}\right]$ is $P_{4}$-free and every component of $G\left[W_{i} \cup W_{i+2}\right]$ is a complete bipartite graph.

If $G\left[W_{i} \cup W_{i+2}\right]$ contains a $P_{4}$, say on vertices $w, x, y, z$ then $G\left[v_{i+3}, v_{i+4}, w, x, y, z\right]$ is a $P_{2}+P_{4}$, a contradiction. Therefore $G\left[W_{i} \cup W_{i+2}\right]$ is indeed $P_{4}$-free. By Claim $1, W_{i}$ and $W_{i+2}$ are independent. Therefore $G\left[W_{i} \cup W_{i+2}\right]$ is a $P_{4}$-free bipartite graph, so every component of $G\left[W_{i} \cup W_{i+2}\right]$ is a complete bipartite graph. This completes the proof of Claim 16 .

Note that $W_{i}$ and $W_{i+2}$ are independent sets by Claim 1, and Claim 16 implies that every component of $G\left[W_{i} \cup W_{i+2}\right]$ is a complete bipartite graph. Suppose $X$ and $Y$ are independent sets such that every component of $G[X \cup Y]$ is a complete bipartite graph. We say that a pair $\{X, Y\}$ is non-simple or that $X$ is non-simple to $Y$ if the number of non-trivial components (i.e. those containing at least one edge) in $G[X \cup Y]$ is at least two. Otherwise, we will say that the pair $\{X, Y\}$ is simple or that $X$ is simple to $Y$. Note that if $\{X, Y\}$ is simple then all the edges in $G[X \cup Y]$ can be removed by using at most one bipartite complementation.

Claim 17. For $i \in\{1, \ldots, 5\}$, if $W_{i}, W_{i+2}$ and $W_{i-2}$ are all non-empty and $W_{i+2}$ is anti-complete to $W_{i-2}$, then $W_{i}$ is complete to $W_{i+2} \cup W_{i-2}$. Furthermore, in this case for all $j \in\{1, \ldots, 5\}, W_{j}$ is anti-complete to $W_{j+1}$ and complete to $W_{j+2}$.

Suppose $x \in W_{i}, y \in W_{i+2}$ and $z \in W_{i-2}$ and that $W_{i+2}$ is anti-complete to $W_{i-2}$. Suppose, for contradiction, that $x$ is non-adjacent to $y$. Then $G\left[y, v_{i+2}, v_{i-1}, v_{i}, x, z\right]$ or $G\left[x, v_{i}, y, v_{i+2}, v_{i-2}, z\right]$ is a $P_{2}+P_{4}$ if $x$ is adjacent or non-adjacent to $z$, respectively. This contradiction implies that $x$ is adjacent to $y$. We conclude that $W_{i}$ is complete to $W_{i+2}$. By symmetry, $W_{i}$ is also complete to $W_{i-2}$. Therefore the first part of Claim 17 holds.

Now suppose that there is a vertex $w \in W_{i+1}$. Since $W_{i-2}$ is anti-complete to $W_{i+2}$, Claim 15 implies that $W_{i+2}$ is anti-complete to $W_{i+1}$ and so $W_{i+1}$ is anti-complete to $W_{i}$. Applying the first part of Claim 17 , we find that $W_{i+1}$ is complete to $W_{i-2}$ and if $W_{i-1}$ is non-empty then $W_{i+1}$ is complete to $W_{i-1}$. Claim 17 now follows by symmetry.

Claim 18. For $i \in\{1, \ldots, 5\}$, if $\left\{W_{i}, W_{i+2}\right\}$ and $\left\{W_{i-2}, W_{i}\right\}$ are both non-simple, then $W_{i-2}$ is complete to $W_{i+2}$ and $W_{i-1}=$ $W_{i+1}=\emptyset$.

Suppose $W_{i}, W_{i+2}$ and $W_{i-2}$ are all non-empty and $\left\{W_{i}, W_{i+2}\right\}$ is non-simple. By Claim $17, W_{i-2}$ is not anti-complete to $W_{i+2}$, so by Claim $14, W_{i-2}$ is complete to $W_{i+2}$. Now suppose, for contradiction, that $W_{i+1}$ contains a vertex $x$. By Claim 15 , since $W_{i-2}$ is complete to $W_{i+2}$, it follows that $W_{i+2}$ is complete to $W_{i+1}$ and therefore $W_{i+1}$ is complete 
to $W_{i}$. Since $\left\{W_{i}, W_{i+2}\right\}$ is not simple, there must be adjacent vertices $y \in W_{i}$ and $z \in W_{i+2}$. Now $G[x, y, z]$ is a $K_{3}$, a contradiction. Claim 18 follows by symmetry.

Claim 19. G contains at most two non-simple pairs, and if it contains two, they must be as described in Claim 18, namely these two pairs must be $\left\{W_{i}, W_{i+2}\right\}$ and $\left\{W_{i-2}, W_{i}\right\}$ for some $i \in\{1, \ldots, 5\}$.

Suppose, for contradiction, that the claim is false. Then for some $i \in\{1, \ldots, 5\}$ both $\left\{W_{i}, W_{i+2}\right\}$ and $\left\{W_{i-1}, W_{i+1}\right\}$ must be non-simple pairs. Thus, by Claim $17, W_{i}$ cannot be anti-complete to $W_{i-1}$, so by Claim $14, W_{i}$ is complete to $W_{i-1}$. Thus, by Claim 15, it follows that $W_{i}$ is complete to $W_{i+1}$. Since $\left\{W_{i-1}, W_{i+1}\right\}$ is non-simple, there must be adjacent vertices $x \in W_{i-1}$ and $z \in W_{i+1}$. Choosing an arbitrary $y \in W_{i}$, we find that $G[x, y, z]$ is a $K_{3}$, a contradiction. This completes the proof of Claim 19.

If $\left\{W_{i}, W_{i+2}\right\}$ is a non-simple pair, then let $W_{i}^{+}$be the set of vertices in $W_{i}$ that have neighbours in $W_{i+2}$ and let $W_{i+2}^{-}$ be the set of vertices in $W_{i+2}$ that have neighbours in $W_{i}$; note that in this case $W_{i} \backslash W_{i}^{+}$is anti-complete to $W_{i+2} \backslash W_{i+2}^{-}$. If $\left\{W_{i}, W_{i+2}\right\}$ is a simple pair, then set $W_{i}^{+}=W_{i+2}^{-}=\emptyset$. Let $W^{*}=\bigcup W_{i}^{+} \cup \cup W_{i}^{-}$and note that $\left\{W_{i} \backslash W^{*}, W_{i+2} \backslash W^{*}\right\}$ is a simple pair in $G \backslash\left(U \cup V^{*} \cup W^{*}\right)$. Next, we look at the edges from the sets $W_{i}^{+}$and $W_{i+2}^{-}$to the remainder of $G \backslash\left(U \cup V^{*}\right)$ and show how we could apply bipartite complementations to remove these sets from the graph.

Claim 20. For $i \in\{1, \ldots, 5\}, W_{i}^{+} \cap W_{i}^{-}=\emptyset$.

Indeed, suppose for contradiction that there is a vertex $x \in W_{i}^{+} \cap W_{i}^{-}$. Then $\left\{W_{i}, W_{i+2}\right\}$ and $\left\{W_{i-2}, W_{i}\right\}$ must be nonsimple pairs and we can choose $y \in W_{i-2}$ and $z \in W_{i+2}$ that are adjacent to $x$. By Claim $18, W_{i-2}$ is complete to $W_{i+2}$. Thus $G[x, y, z]$ is a $K_{3}$, a contradiction. Claim 20 follows.

Claim 21. For $i \in\{1, \ldots, 5\}$, suppose $\left\{W_{i}, W_{i+2}\right\}$ is a non-simple pair. Then $V_{i+2}$ is complete to $W_{i+2}$ and anti-complete to $W_{i}^{+}$. Similarly, $V_{i}$ is complete to $W_{i}$ and anti-complete to $W_{i+2}^{-}$.

Suppose, for contradiction, that $x \in V_{i+2}$ has a non-neighbour $z \in W_{i+2}$. Since $\left\{W_{i}, W_{i+2}\right\}$ is non-simple, there is a vertex $y \in W_{i}$ that is non-adjacent to $z$. Now $G\left[v_{i+2}, z, x, y, v_{i}, v_{i-1}\right]$ or $G\left[v_{i}, y, z, v_{i+2}, v_{i+3}, x\right]$ is a $P_{2}+P_{4}$ is $x$ is adjacent or non-adjacent to $y$, respectively. This contradiction implies that $V_{i+2}$ is complete to $W_{i+2}$.

Now suppose, for contradiction, that $x \in V_{i+2}$ has a neighbour $y \in W_{i}^{+}$. Since $y \in W_{i}^{+}$it must have a neighbour $z \in$ $W_{i+2}$ and by the previous paragraph, $x$ must be adjacent to $z$. Now $G[x, y, z]$ is a $K_{3}$, a contradiction. Therefore $V_{i+2}$ is anti-complete to $W_{i}^{+}$. Claim 21 follows by symmetry.

Claim 22. For $i \in\{1, \ldots, 5\}$, suppose $\left\{W_{i}, W_{i+2}\right\}$ is a non-simple pair. Then $V_{i+3}$ is anti-complete to $W_{i+2}$ and every vertex of $V_{i+3}$ is either anti-complete to $W_{i}$ or complete to $W_{i}^{+}$. Similarly, $V_{i+4}$ is anti-complete to $W_{i}$ and every vertex of $V_{i+4}$ is either anti-complete to $W_{i+2}$ or complete to $W_{i+2}^{-}$.

By Claim 2, $W_{i+2}$ is anti-complete to $V_{i+3}$. Suppose, for contradiction, that the claim does not hold for some vertex $x \in V_{i+3}$. Then $x$ must have a neighbour $y \in W_{i}$ and a non-neighbour $y^{\prime} \in W_{i}^{+}$and since $\left\{W_{i}, W_{i+2}\right\}$ is non-simple, we may assume that $y$ and $y^{\prime}$ are in different components of $G\left[W_{i} \cup W_{i+2}\right.$. Indeed, if $y$ and $y^{\prime}$ are in the same component, then there must be a vertex $y^{\prime \prime} \in W_{i}^{+}$in a different component of $G\left[W_{i} \cup W_{i+2}\right]$ to $y$ and $y^{\prime}$; in this case we replace $y$ or $y^{\prime}$ by $y^{\prime \prime}$ if $x$ is adjacent or non-adjacent to $y^{\prime \prime}$, respectively. Since $y^{\prime} \in W_{i}^{+}$it must have a neighbour $z^{\prime} \in W_{i+2}$ and then $z^{\prime}$ is non-adjacent to $y$. Now $G\left[y^{\prime}, z^{\prime}, y, x, v_{i+4}, v_{i+3}\right]$ is a $P_{2}+P_{4}$, a contradiction. Claim 22 follows by symmetry.

Claim 23. For $i \in\{1, \ldots, 5\}$, every vertex of $V(G) \backslash\left(V^{*} \cup U \cup W_{i}^{+} \cup W_{i+2}^{-}\right)$is either complete or anti-complete to $W_{i}^{+}$and either complete or anti-complete to $W_{i+2}^{-}$.

We may assume that $\left\{W_{i}, W_{i+2}\right\}$ is non-simple, otherwise $W_{i}^{+}$and $W_{i+2}^{-}$are empty and the claim holds trivially. By symmetry it is enough to show that every vertex of $V(G) \backslash\left(V^{*} \cup U \cup W_{i}^{+} \cup W_{i+2}^{-}\right)$is either complete or anti-complete to $W_{i}^{+}$. First note that $v_{i}$ is complete to $W_{i}^{+}$and $V(C) \backslash\left\{v_{i}\right\}$ is anti-complete to $W_{i}^{+}$by definition of $W_{i}$. By Claim 2, every vertex of $V_{i+1}^{0}$ is anti-complete to $W_{i}^{+}$. By Claim 21, every vertex of $V_{i}^{0}$ is complete to $W_{i}^{+}$and every vertex of $V_{i+2}^{0}$ is anti-complete to $W_{i}^{+}$. By Claim 22, every vertex of $V_{i+3}^{0}$ and $V_{i+4}^{0}$ is either complete or anti-complete to $W_{i}^{+}$. By Claim 1 , every vertex of $W_{i} \backslash W_{i}^{+}$is anti-complete to $W_{i}^{+}$. By Claim 14, every vertex of $W_{i-1}$ and $W_{i+1}$ is either complete or anti-complete to $W_{i}^{+}$. By definition of $W_{i}^{+}$, every vertex of $W_{i+2} \backslash W_{i+2}^{-}$is anti-complete to $W_{i}^{+}$. Suppose, for contradiction, that a vertex $x \in W_{i-2}$ has a neighbour $y \in W_{i}^{+}$. Since $\left\{W_{i}, W_{i+2}\right\}$ is non-simple, Claim 17 implies that $W_{i-2}$ is not anti-complete to $W_{i+2}$, so by Claim $14, W_{i-2}$ is complete to $W_{i+2}$. Since $y \in W_{i}^{+}$there must be a vertex $z \in V_{i+2}$ that 
is adjacent to $y$. Now $G[x, y, z]$ is a $K_{3}$, a contradiction. Therefore $W_{i-2}$ is anti-complete to $W_{i}^{+}$. Claim 23 follows by symmetry.

\section{Claim 24. Every module $M$ in $G \backslash\left(U \cup V^{*} \cup W^{*}\right)$ that is an independent set contains at most 512 vertices.}

Recall that $G$ is prime, so it contains no non-trivial modules. Let $H=G \backslash\left(V^{*} \cup U \cup W^{*}\right)$ and suppose, for contradiction, that $H$ contains a module $M$ on at least 513 vertices such that $M$ is an independent set. By Claim 19 all but at most two sets of the form $W_{i}^{+}$are empty and all but at most two sets of the form $W_{i}^{-}$are empty in $G$. By Claim 23, for all $i \in\{1, \ldots, 5\}$, every vertex of $M$ is either complete or anti-complete to $W_{i}^{+}$and $W_{i}^{-}$in $G$. By Claim 9 , for all $i \in\{1, \ldots, 5\}$, every vertex of $M$ is either complete or anti-complete to $V_{i}^{*}$ in $G$ and every vertex of $M$ is anti-complete to $U$ in $G$. Therefore in $G$ every vertex of $M$ has one of at most $2^{(4+5)}=512$ neighbourhoods in $U \cup V^{*} \cup W^{*}$. Choose two vertices $x, y \in M$ that have the same neighbourhood in $U \cup V^{*} \cup W^{*}$. Since $M$ is a module in $H, x$ and $y$ are not distinguished by any vertex of $V(H) \backslash M$. Since $M$ is an independent set, $x$ and $y$ are not distinguished by any vertex of $M \backslash\{x, y\}$. Therefore $\{x, y\}$ is a non-trivial module in $G$. This contradiction completes the proof of Claim 24.

\section{Claim 25. We may assume that $U \cup V^{*} \cup W^{*}=\emptyset$.}

By Claim 9, we may apply at most five bipartite complementations to separate $G\left[U \cup V^{*}\right]$ from the rest of the graph. Now, by Claim 13, we can delete at most five vertices from $G\left[U \cup V^{*}\right]$ to obtain a graph that is either bipartite (in which case it is curious) or 3-uniform. Suppose that $G$ contains a non-simple pair $\left\{W_{i}, W_{i+2}\right\}$ and let $H=G \backslash\left(V^{*} \cup U \cup W_{i}^{+} \cup W_{i+2}^{-}\right)$. Then Claim 23 shows that we can remove all edges between $W_{i}^{+} \cup W_{i+2}^{-}$and the vertices of $H$ by applying at most two bipartite complementations. Furthermore, $G\left[W_{i}^{+} \cup W_{i+2}^{-}\right]$is a bipartite graph by Claim 1 , so it is a curious $\left(K_{3}, P_{2}+P_{4}\right)$-free graph with one part of the 3-partition empty. By Claim 19, $G$ contains at most two non-simple pairs and if it contains two then by Claim 20, $W_{i}^{+} \cap W_{i}^{-}=\emptyset$ for all $i$. Hence, Claim 23 implies that by applying at most four bipartite complementations, we can separate $G\left[W^{*}\right]$ from the rest of $G \backslash\left(V^{*} \cup U\right)$. We do this by separating the curious graph $G\left[W_{i}^{+} \cup W_{i+2}^{-}\right]$for each non-simple pair $\left\{W_{i}, W_{i+2}\right\}$ in turn. We may therefore assume that $U \cup V^{*} \cup W^{*}=\emptyset$. This completes the proof of Claim 25.

From now on, we assume that $U=\emptyset$ and $\left\{W_{i}, W_{i+2}\right\}$ is simple for every $i \in\{1, \ldots, 5\}$. Indeed, recall that if (prior to the assumption of Claim 25) $\left\{W_{i}, W_{i+2}\right\}$ was a non-simple pair, then $W_{i} \backslash W^{*}$ was anti-complete to $W_{i+2} \backslash W^{*}$ in $G$, so $\left\{W_{i} \backslash W^{*}, W_{i+2} \backslash W^{*}\right\}$ was simple in $G \backslash\left(U \cup V^{*} \cup W^{*}\right)$. Note that in the proof of Claim 25 we edit the graph $G$ in such a way that it may stop being prime. We may do so at this stage in the proof, as the remaining arguments in the proof do not directly rely on the property that $G$ is prime. Instead, we will rely on a weaker property, which follows from Claim 24 and still holds for the edited graph $G$, namely that $G$ does not contain any module $M$ that is an independent set on more than 512 vertices. As we shall see, this weaker property will suffice for completing the proof.

Claim 26. For $i \in\{1, \ldots, 5\}$, in every set of 513 vertices in $V_{i}$, there are two that together dominate either $V_{i+1}$ or $V_{i-1}$.

Let $X \subseteq V_{i}$ with $|X| \geq 513$ and note that $X$ is an independent set by Claim 2. By Claim 24, $X$ cannot be a module, so there must be two vertices $x, y \in X$ that are distinguished by a vertex $z$ outside $X$, say $z$ is adjacent to $x$, but non-adjacent to $y$. By definition of $V_{i}$, vertices in the cycle $C$ cannot distinguish two vertices in the same set $V_{i}$, so $z \notin C$. We will show that $\{x, y\}$ dominates either $V_{i+1}$ or $V_{i-1}$. Suppose, for contradiction, that $x$ and $y$ are both non-adjacent to $a \in V_{i-1}$ and both non-adjacent to $b \in V_{i+1}$. By Claim 2 vertices in $V_{i-2} \cup V_{i+2} \cup W_{i-1} \cup W_{i+1} \cup\left(V_{i} \backslash\{x, y\}\right)$ are anti-complete to $\{x, y\}$. By symmetry, we may therefore assume that $z \in V_{i+1} \cup W_{i} \cup W_{i+2}$. In this case $z$ is non-adjacent to $v_{i+1}, v_{i-1}$ and $v_{i-2}$. Now $z$ must be adjacent to $a$, otherwise $G\left[a, v_{i-2}, z, x, v_{i+1}, y\right]$ would be a $P_{2}+P_{4}$. Now $z$ cannot belong to $V_{i+1} \cup W_{i}$, otherwise $G\left[a, v_{i}, z\right]$ would be a $K_{3}$. Therefore $z \in W_{i+2}$. Now $z$ cannot be adjacent to $b$ otherwise $G\left[v, v_{i+2}, z\right]$ would be a $K_{3}$ and by Claim 2, $a$ is non-adjacent to $b$. It follows that $G\left[y, v_{i-1}, a, z, v_{i+2}, b\right]$ is a $P_{2}+P_{4}$. This contradiction completes the proof of Claim 26.

Claim 27. By deleting at most 512 vertices from each set $V_{i}$ we may assume that each vertex of $V_{i}$ dominates either $V_{i-1}$ or $V_{i+1}$.

Note that $V_{i-1}$ is anti-complete to $V_{i+1}$ by Claim 2. For each $x \in V_{i-1}$ and $y \in V_{i+1}$, let $A^{x, y}$ denote the set of vertices in $V_{i}$ that are non-adjacent to both $x$ and $y$. Consider an $x \in V_{i-1}$ and a $y \in V_{i+1}$ such that $A^{x, y}$ is non-empty, say $a \in A^{x, y}$. (Note that if $A^{x, y}$ is empty for all $x$ and $y$ then we are done.) If a vertex $b \in V_{i}$ is adjacent to exactly one of $x$ and $y$, say $b$ is adjacent to $x$, then $G\left[v_{i+2}, y, a, v_{i-1}, b, x\right]$ is a $P_{2}+P_{4}$, a contradiction. This contradiction shows that if $A^{x, y}$ is non-empty then $N(x) \cap V_{i}=N(y) \cap V_{i}=V_{i} \backslash A^{x, y}$.

Now choose $x$ and $y$ such that $\left|A^{x, y}\right|$ is maximum. Suppose, for contradiction, that there is a vertex $b \in V_{i} \backslash A^{x, y}$ with a non-neighbour $x^{\prime} \in V_{i-1}$ and a non-neighbour $y^{\prime} \in V_{i+1}$. Note that $b$ must be adjacent to both $x$ and $y$ and $b \in A^{x^{\prime}, y^{\prime}}$. Furthermore, since $\left|A^{x, y}\right|$ is maximum, there must be a vertex $a \in A^{x, y} \backslash A^{x^{\prime}, y^{\prime}}$, so $a$ is adjacent to both $x^{\prime}$ and $y^{\prime}$. Now $G\left[a, x^{\prime}, x, b, y, v_{i+2}\right]$ is a $P_{2}+P_{4}$, a contradiction. It follows that every vertex in $V_{i} \backslash A^{x, y}$ dominates either $V_{i-1}$ or $V_{i+1}$. By Claim 26, $\left|A^{x, y}\right| \leq 512$, so we delete the vertices of $A^{x, y}$. This completes the proof of Claim 27. 
By Claim 27, we may assume that every vertex of $V_{i}$ dominates either $V_{i-1}$ or $V_{i+1}$. Therefore, we can partition each set $V_{i}$ into three (possibly empty) subsets as follows:

- $V_{i}^{0}$ the set of vertices in $V_{i}$ that dominate both $V_{i-1}$ and $V_{i+1}$,

- $V_{i}^{-}$the set of vertices in $V_{i} \backslash V_{i}^{0}$ that dominate $V_{i-1}$ (and so have non-neighbours in $V_{i+1}$ ),

- $V_{i}^{+}$the set of vertices in $V_{i} \backslash V_{i}^{0}$ that dominate $V_{i+1}$ (and so have non-neighbours in $V_{i-1}$ ).

By definition of this partition, if $x \in V_{i}$ is non-adjacent to $y \in V_{i+1}$, then $x \in V_{i}^{-}$and $y \in V_{i+1}^{+}$. Moreover, every vertex of $V_{i}^{-}$ has a non-neighbour in $V_{i+1}^{+}$and vice versa. Thus, the vertices of $V_{1} \cup \cdots \cup V_{5}$ are partitioned into 15 subsets (some or all of which may be empty). Note that for $i \in\{1, \ldots, 5\}$ the sets $V_{i}^{0}, V_{i}^{-}, V_{i}^{+}, V_{i+2}^{0}, V_{i+2}^{-}$and $V_{i+2}^{+}$are pairwise anti-complete by Claim 2. Furthermore, for $i \in\{1, \ldots, 5\}, V_{i}^{0}$ and $V_{i}^{+}$are complete to $V_{i+1}^{0}, V_{i+1}^{-}$and $V_{i+1}^{+}$, and $V_{i+1}^{-}$is complete to $V_{i}^{0}$ and $V_{i}^{-}$by definition. Therefore nearly every pair of these subsets is either complete or anti-complete to each other. The only possible exceptions are the five disjoint pairs of the form $\left\{V_{i}^{-}, V_{i+1}^{+}\right\}$.

Recall that for two independent sets of vertices $X$ and $Y$ such that $G[X \cup Y]$ is $P_{4}$-free, the components of $G[X \cup Y]$ are complete bipartite graphs and if $G[X \cup Y]$ contains at most one non-trivial component, we say that the pair $\{X, Y\}$ is simple and otherwise it is non-simple. Recall that if $\{X, Y\}$ is simple then we can remove all edges between $X$ and $Y$ by applying at most one bipartite complementation. By Claim 14 every pair $\left\{W_{i}, W_{i+1}\right\}$ is simple and by Claim 25 we have assumed that the set $W^{*}$ is empty, so every pair $\left\{W_{i}, W_{i+2}\right\}$ is simple. Therefore every pair $\left\{W_{i}, W_{j}\right\}$ is simple and for the fifteen sets considered in the previous paragraph, the only pairs of them that can be non-simple are the ones of the form $\left\{V_{i}^{-}, V_{i+1}^{+}\right\}$.

Next, we will analyse the edges between sets of the form $W_{i}$ and sets of the form $V_{j}$ (and their subsets $V_{j}^{0}, V_{j}^{-}$ and $V_{j}^{+}$) for $i, j \in\{1, \ldots, 5\}$. We will then use bipartite complementations to partition the graph into induced subgraphs that are curious. First, recall that $W_{i}$ is anti-complete to $V_{i-1}$ and $V_{i+1}$ by Claim 2. Also, by Claim 4 , every vertex of $V_{i}$ is either complete or anti-complete to $W_{i}$. Since $V_{i}$ and $W_{i}$ are independent sets by Claim 2 , it follows that $G\left[V_{i} \cup W_{i}\right]$ contains at most one non-trivial component, so $\left\{W_{i}, V_{i}\right\}$ is simple. Therefore, the only sets $V_{j}$ with which $W_{i}$ can form a non-simple pair are $V_{i+2}$ and $V_{i+3}$.

Claim 28. For $i \in\{1, \ldots, 5\}$, if $x \in W_{i}, y \in V_{i+2}$ and $z \in V_{i+3}$, then $G[x, y, z]$ is not a $3 P_{1}$.

Indeed, if $x \in W_{i}, y \in V_{i+2}$ and $z \in V_{i+3}$ and $G[x, y, z]$ is a $3 P_{1}$ then $G\left[x, v_{i}, y, v_{i+3}, v_{i+2}, z\right]$ is a $P_{2}+P_{4}$, a contradiction. This completes the proof of Claim 28.

Claim 29. For $i \in\{1, \ldots, 5\}$ :

(i) If $x \in W_{i}$ has a neighbour in $V_{i+2}^{0} \cup V_{i+2}^{+}$, then $x$ is anti-complete to $V_{i+3}$ and complete to $V_{i+2}^{-}$.

(ii) If $x \in W_{i}$ has a neighbour in $V_{i+3}^{0} \cup V_{i+3}^{-}$, then $x$ is anti-complete to $V_{i+2}$ and complete to $V_{i+3}^{+}$.

Suppose $x \in W_{i}$ has a neighbour $y \in V_{i+2}^{0} \cup V_{i+2}^{+}$. If $z \in V_{i+3}$ then $y$ is adjacent to $z$, so $x$ must be non-adjacent to $z$, otherwise $G[x, y, z]$ would be a $K_{3}$. Therefore $x$ is anti-complete to $V_{i+3}$. If $y^{\prime} \in V_{i+2}^{-}$then $y^{\prime}$ has a non-neighbour $z^{\prime} \in V_{i+3}$. Note that $z^{\prime}$ is non-adjacent to $x$. Now $x$ must be adjacent to $y^{\prime}$, otherwise $G\left[x, y^{\prime}, z^{\prime}\right]$ would be a $3 P_{1}$, contradicting Claim 28. It follows that $x$ is complete to $V_{i+2}^{-}$. Claim 29 follows by symmetry.

By Claim 29 we can partition $W_{i}$ into three (possibly empty) subsets as follows:

- $W_{i}^{2}$ the set of vertices in $W_{i}$ that have neighbours in $V_{i+2}^{0} \cup V_{i+2}^{+}$(and are therefore anti-complete to $V_{i+3}$ and complete to $\left.V_{i+2}^{-}\right)$,

- $W_{i}^{3}$ the set of vertices in $W_{i}$ that have neighbours in $V_{i+3}^{0} \cup V_{i+3}^{-}$(and are therefore anti-complete to $V_{i+2}$ and complete to $\left.V_{i+3}^{+}\right)$and

- $W_{i}^{0}$ the set of vertices in $W_{i} \backslash\left(W_{i}^{2} \cup W_{i}^{3}\right.$ ) (which are therefore anti-complete to $V_{i+2}^{0} \cup V_{i+2}^{+} \cup V_{i+3}^{0} \cup V_{i+3}^{-}$).

Recall that the only sets $V_{j}$ with which $W_{i}$ can have form a non-simple pair are $V_{i+2}$ and $V_{i+3}$. Therefore, by Claim 29 , for $i, j \in\{1, \ldots, 5\}$ if a set in $\left\{W_{i}^{2}, W_{i}^{3}, W_{i}^{0}\right\}$ is non-simple to a set in $\left\{V_{j}^{-}, V_{j}^{+}, V_{j}^{0}\right\}$ then it must be one of the following pairs:

$$
\begin{aligned}
& -\left\{W_{i}^{2}, V_{i+2}^{0}\right\}, \\
& -\left\{W_{i}^{2}, V_{i+2}^{+}\right\}, \\
& -\left\{W_{i}^{3}, V_{i+3}^{0}\right\},
\end{aligned}
$$




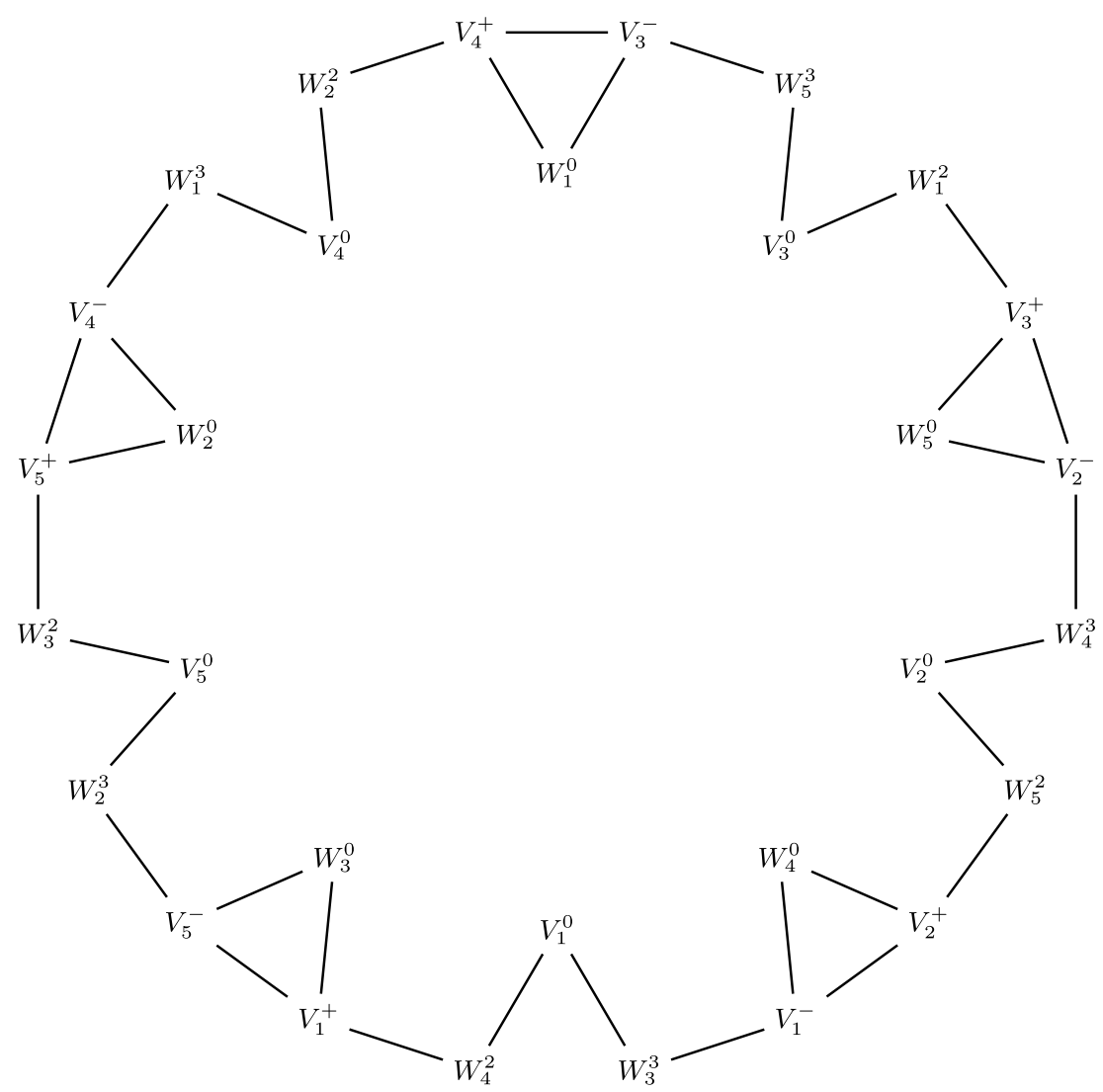

Fig. 4. The connections between pairs of sets of the form $V_{j}^{-}, V_{j}^{+}, V_{j}^{0}, W_{j}^{2}, W_{j}^{3}$ and $W_{j}^{0}$. An edge is shown between two such sets if it is possible for this pair of sets to be non-simple. If an edge is not shown between two sets, then these sets form a simple pair and therefore all edges between them can be removed by using at most one bipartite complementation.

$-\left\{W_{i}^{3}, V_{i+3}^{-}\right\}$,

$-\left\{W_{i}^{0}, V_{i+2}^{-}\right\}$or

$-\left\{W_{i}^{0}, V_{i+3}^{+}\right\}$.

Recall among pairs of sets of the form $V_{j}^{-}, V_{j}^{+}, V_{j}^{0}, W_{j}^{2}, W_{j}^{3}, W_{j}^{0}$ the only possible other non-simple pairs are the ones of the form $\left\{V_{i}^{-}, V_{i+1}^{+}\right\}$(see also Fig. 4).

Notice that $W_{i}^{2}$ and $W_{i}^{3}$ are disjoint by Claim 29. This partition allows us to distinguish two kinds of induced subgraphs of $G$.

1. If for some $i \in\{1, \ldots, 5\} W_{i}^{3}$ and $W_{i+1}^{2}$ are both non-empty then we form a subgraph of the first kind $H_{i}^{1}=G\left[W_{i}^{3} \cup\right.$ $W_{i+1}^{2} \cup V_{i+3}^{0}$ ]. (If $W_{i}^{3}$ or $W_{i+1}^{2}$ is empty, then we let $H_{i}^{1}$ be the empty graph $(\emptyset, \emptyset)$.)

2. For $i \in\{1, \ldots, 5\}$ we let $H_{i}^{2}$ be the graph induced on $G$ by $W_{i}^{0} \cup V_{i+2}^{-} \cup V_{i+3}^{+}$. If $H_{i-1}^{1}$ is empty, but $W_{i-1}^{3}$ is non-empty then $W_{i}^{2}=\emptyset$. In this case we also add $W_{i-1}^{3} \cup V_{i+2}^{0}$ to $H_{i}^{2}$. Similarly, if $H_{i}^{1}$ is empty, but $W_{i+1}^{2}$ is non-empty then $W_{i}^{3}=\emptyset$. In this case we also add $W_{i+1}^{2} \cup V_{i+3}^{0}$ to $H_{i}^{2}$. We say that $H_{i}^{2}$ is a graph of the second kind.

In the next two claims, we show how to disconnect both kinds of graph $H_{i}^{j}$ from the rest of the graph.

Claim 30. For $i \in\{1, \ldots, 5\}$ the graph $H_{i}^{1}$ can be separated from the rest of the graph using finitely many bipartite complementations.

We will show that for every set $V_{j}^{-}, V_{j}^{+}, V_{j}^{0}, W_{j}^{2}, W_{j}^{3}, W_{j}^{0}$ that is not in $\left\{W_{i}^{3}, W_{i+1}^{2}, V_{i+3}^{0}\right\}$, this set is simple to each of the three sets in $\left\{W_{i}^{3}, W_{i+1}^{2}, V_{i+3}^{0}\right\}$, in which case we can separate $H_{i}^{1}$ by applying at most one bipartite complementation between every pair of such sets. Taking into account Claim 29, recall that $W_{i}^{3}$ is simple to every set except $V_{i+3}^{0}$ and $V_{i+3}^{-}$, $W_{i+1}^{2}$ is simple to every set except $V_{i+3}^{0}$ and $V_{i+3}^{+}$and $V_{i+3}^{0}$ is simple to every set except $W_{i}^{3}$ and $W_{i+1}^{2}$. Thus, it suffices to 
show that $W_{i}^{3}$ is simple to $V_{i+3}^{-}$and $W_{i+1}^{2}$ is simple to $V_{i+3}^{+}$. By symmetry, we need only show that $W_{i+1}^{2}$ is simple to $V_{i+3}^{+}$. If $W_{i+1}^{2}$ is anti-complete to $V_{i+3}^{+}$then we are done. Otherwise, choose a vertex $x \in W_{i+1}^{2}$ that has a neighbour $y \in V_{i+3}^{+}$. Note that $W_{i}^{3}$ is non-empty, by assumption (otherwise $H_{i}^{1}$ is empty and we are done). By Claim 29, $V_{i+3}^{+}$is complete to $W_{i}^{3}$. Therefore $x$ must be anti-complete to $W_{i}^{3}$, otherwise for $z \in W_{i}^{3}, G[x, y, z]$ would be a $K_{3}$, a contradiction. We will show that $x$ is complete to $V_{i+3}^{+}$. Suppose, for contradiction, that $x$ has a non-neighbour $y^{\prime} \in V_{i+3}^{+}$. By definition of $V_{i+3}^{+}$and $V_{i+2}^{-}$, $y^{\prime}$ must have a non-neighbour $z^{\prime} \in V_{i+2}^{-}$. Let $u \in W_{i}^{3}$ and note that $u$ is non-adjacent to $z^{\prime}$ and adjacent to $y^{\prime}$ by Claim 29 . Thus $G\left[u, y^{\prime}, x, v_{i+1}, z^{\prime}, v_{i+3}\right]$ is a $P_{2}+P_{4}$. This contradiction shows that if $x \in W_{i+1}^{2}$ has a neighbour in $V_{i+3}^{+}$then $x$ is complete to $V_{i+3}^{+}$. Therefore, $\left\{W_{i+1}^{2}, V_{i+3}^{+}\right\}$is simple. Similarly, $\left\{W_{i}^{3}, V_{i+3}^{-}\right\}$is simple. Applying bipartite complementations between $W_{i}^{3} \&\left\{v_{i}\right\}, W_{i+1}^{2} \&\left\{v_{i+1}\right\}$ and $V_{i+3}^{0} \&\left\{v_{i+2}, v_{i+4}\right\}$ removes all edges between the vertices of $H_{i}^{1}$ and the vertices of the cycle $C$. Every set $V_{j}^{-}, V_{j}^{+}, V_{j}^{0}, W_{j}^{2}, W_{j}^{3}, W_{j}^{0}$ for $j \in\{1, \ldots, 5\}$ that is not in $\left\{W_{i}^{3}, W_{i+1}^{2}, V_{i+3}^{0}\right\}$ is simple to every set in $\left\{W_{i}^{3}, W_{i+1}^{2}, V_{i+3}^{0}\right\}$, so applying at most one bipartite complementation between each such pair of sets, we can remove all remaining edges from $H_{i}^{1}$ to the rest of the graph. This completes the proof of Claim 30.

Claim 31. For $i \in\{1, \ldots, 5\}$ the graph $H_{i}^{2}$ can be separated from the rest of the graph using finitely many bipartite complementations.

The graph $H_{i}^{2}$ contains $W_{i}^{0} \cup V_{i+2}^{-} \cup V_{i+3}^{+}$. Taking into account Claim 29, recall that the set $W_{i}^{0}$ can only be non-simple to $V_{i+2}^{-}$or $V_{i+3}^{+}$, the set $V_{i+2}^{-}$can only be non-simple to $V_{i+3}^{+}, W_{i}^{0}$ and $W_{i-1}^{3}$, and the set $V_{i+3}^{+}$can only be non-simple to $V_{i+2}^{-}, W_{i}^{0}$ and $W_{i+1}^{2}$. Thus we only need to concern ourselves with the case where the pair $\left\{V_{i+2}^{-}, W_{i-1}^{3}\right\}$ or the pair $\left\{V_{i+3}^{+}, W_{i+1}^{2}\right\}$ is not simple. By symmetry, it is enough to consider the second of these cases. If $W_{i+1}^{2}$ is in the graph $H_{i}^{1}$ then we are done by Claim 30. Therefore we may assume that $W_{i+1}^{2}$ is non-empty, but not contained in the graph $H_{i}^{1}$, so $W_{i}^{3}=\emptyset$. Thus we add $W_{i+1}^{2} \cup V_{i+3}^{0}$ to $H_{i}^{2}$. Now $W_{i+1}^{2}$ can only be non-simple to $V_{i+3}^{0}$ and $V_{i+3}^{+}$, both of which are in $H_{i}^{2}$ and $V_{i+3}^{0}$ can only be non-simple to $W_{i+1}^{2}$, which is in $H_{i}^{2}$ and $W_{i}^{3}$, which is empty. By symmetry, it follows that we can use a bounded number of bipartite complementations to disconnect $H_{i}^{2}$ from all sets of the form $V_{j}^{-}, V_{j}^{+}, V_{j}^{0}, W_{j}^{2}, W_{j}^{3}$, $W_{j}^{0}$ outside $H_{i}^{2}$. Finally, applying a bipartite complementation between each of the sets in $H_{i}^{2}$ and their neighbourhood in the cycle $C$ disconnects $H_{i}^{2}$ from the remainder of the graph. This completes the proof of Claim 31.

In the next two claims, we show that graphs of the first kind are curious and graphs of the second kind can be partitioned into two curious induced subgraphs by applying at most two bipartite complementations.

Claim 32. For $i \in\{1, \ldots, 5\}$, the graph $H_{i}^{1}$ is bipartite and therefore curious.

The sets $W_{i}^{3}, W_{i+1}^{2}$ and $V_{i+3}^{0}$ are independent by Claim 2. By Claim $14, W_{i}^{3}$ is complete or anti-complete to $W_{i+1}^{2}$. If $W_{i}^{3}$ is anti-complete to $W_{i+1}^{2}$, then $W_{i}^{3} \cup W_{i+1}^{2}$ and $V_{i+3}^{0}$ are independent sets, so $H_{i}^{1}$ is bipartite. If $W_{i}^{3}$ is complete to $W_{i+1}^{2}$, then every vertex of $V_{i+3}^{0}$ has a neighbour in at most one of $W_{i}^{3}$ and $W_{i+1}^{2}$ (since $G$ is $K_{3}$-free), and hence $H_{i}^{1}$ is again bipartite. Since bipartite graphs are curious graphs where one of the partition classes is empty, this completes the proof of Claim 32.

Claim 33. For $i \in\{1, \ldots, 5\}$, it is sufficient to apply at most two bipartite complementation operations to $H_{i}^{2}$ to modify $H_{i}^{2}$ into the disjoint union of at most two curious induced subgraphs of $\mathrm{H}_{i}^{2}$.

The sets $W_{i}^{0}, V_{i+2}^{-}$and $V_{i+3}^{+}$are independent by Claim 2. If $H_{i}^{2}$ only contains the vertices in $W_{i}^{0} \cup V_{i+2}^{-} \cup V_{i+3}^{+}$then it is curious by Claim 28. We may therefore assume that $W_{i-1}^{3}$ and/or $W_{i+1}^{2}$ belong to $H_{i}^{2}$. Note that $X=\left(W_{i-1}^{3} \cup V_{i+3}^{0} \cup\right.$ $\left.V_{i+3}^{+}\right) \cap V\left(H_{i}^{2}\right)$ and $Y=\left(W_{i+1}^{2} \cup V_{i+2}^{0} \cup V_{i+2}^{-}\right) \cap V\left(H_{i}^{2}\right)$ are independent sets by Claim 2. Therefore if $W_{i}^{0}=\emptyset$ then $H_{i}^{2}$ is bipartite, so it is a curious graph, where one part of the 3-partition is empty. If $W_{i}^{0}$ is not empty, then it is either complete or anti-complete to $W_{i-1}^{3} \cup W_{i+1}^{2}$ by Claim 15 .

If $W_{i}^{0}$ is complete to $W_{i-1}^{3} \cup W_{i+1}^{2}$ then taking $X$ and $Y$ as above, and setting $Z=W_{i}^{0}$, we find that $H_{i}^{2}$ is a curious graph, with partition $X, Y, Z$. Indeed, suppose for contradiction that there are vertices $x \in X, y \in Y$ and $z \in Z$ with $G[x, y, z]$ forming a $3 P_{1}$. Since $z \in W_{i}^{0}$, it follows that $x \notin W_{i-1}^{3}$ and $y \notin W_{i+1}^{2}$. Therefore we have $x \in V_{i+2}, y \in V_{i+3}$ and $z \in W_{i}$ such that $G[x, y, z]$ is a $3 P_{1}$, which contradicts Claim 28. This contradiction shows that if $W_{i}^{0}$ is complete to $W_{i-1}^{3} \cup W_{i+1}^{2}$ then $H_{i}^{2}$ is indeed a curious graph.

Finally, suppose that $W_{i}^{0}$ is anti-complete to $W_{i-1}^{3} \cup W_{i+1}^{2}$. By Claim 25, we may assume that $W^{*}$ is empty, and therefore $W_{i-1}^{3}$ is simple to $W_{i+1}^{2}$, so $G\left[W_{i-1}^{3} \cup W_{i+1}^{2}\right]$ contains at most one non-trivial component, and if such a component exists, it must be a complete bipartite graph by Claim 16. Let $W_{i-1}^{3 *}\left(\right.$ resp. $W_{i+1}^{2 *}$ ) be the set of vertices in $W_{i-1}^{3}$ (resp. $W_{i+1}^{2}$ ) 
that have neighbours in $W_{i+1}^{2}$ (resp. $W_{i-1}^{3}$ ). Since $G\left[W_{i-1}^{3 *} \cup W_{i+1}^{2 *}\right]$ is a complete bipartite graph, it follows that $W_{i-1}^{3 *}$ is complete to $W_{i+1}^{2 *}$.

We will show that if $W_{i-1}^{3 *}$ and $W_{i+1}^{2 *}$ are both in $H_{i}^{2}$, then we can disconnect $H_{i}^{2}\left[W_{i-1}^{3 *} \cup W_{i+1}^{2 *}\right]$ from the rest of $H_{i}^{2}$ by applying at most two bipartite complementations. Indeed, suppose $x \in W_{i-1}^{3 *}$ has a neighbour $y \in W_{i+1}^{2 *}$ and let $u \in W_{i}^{0}$. By definition of $W_{i-1}^{3}$ there is a vertex $z \in V_{i+2}^{0} \cup V_{i+2}^{-}$that is adjacent to $x$. Thus $y$ must be non-adjacent to $z$ by Claim 2 . If $z$ is non-adjacent to $u$ then $G\left[v_{i}, u, y, x, z, v_{i-2}\right]$ is a $P_{2}+P_{4}$, a contradiction. Therefore $z$ is adjacent to $u$. By definition of $W_{i}^{0}$, it follows that $z \notin V_{i+2}^{0}$ and so $z \in V_{i+2}^{-}$. We conclude that $W_{i-1}^{3 *}$ is anti-complete to $V_{i+2}^{0}$, and by symmetry, $W_{i+1}^{2 *}$ is anti-complete to $V_{i+3}^{0}$. Now suppose that there is a vertex $z^{\prime} \in V_{i+2}^{-}$such that $x$ is non-adjacent to $z^{\prime}$. If $z^{\prime}$ is adjacent to $u$ then $G\left[x, v_{i-1}, u, z^{\prime}, v_{i+1}, v_{i+2}\right]$ is a $P_{2}+P_{4}$. Therefore $z^{\prime}$ must be non-adjacent to $u$. We conclude that every vertex in $V_{i+2}^{-}$is adjacent to $x$ if and only if it is adjacent to $u$. Since $x$ was chosen from $W_{i-1}^{3 *}$ arbitrarily, this means that every vertex in $V_{i+2}^{-}$is either complete or anti-complete to $W_{i-1}^{3 *}$. Therefore, applying at most one bipartite complementation, we can remove all edges from $V_{i+2}^{-}$to $W_{i-1}^{3 *}$ Recall that $V_{i+2}^{0}$ is anti-complete to $W_{i-1}^{3 *}$. By assumption, $W_{i-1}^{3 *}$ is anti-complete to $W_{i}^{0}$, complete to $W_{i+1}^{2 *}$ and anti-complete to $W_{i+1}^{2} \backslash W_{i+1}^{2 *}$. By Claim $2, W_{i-1}^{3 *}$ is anti-complete to $V_{i+3}$. Therefore, applying at most one bipartite complementation, we can remove all edges between vertices in $W_{i-1}^{3 *}$ and vertices in $V\left(H_{i}^{2}\right) \backslash\left(W_{i-1}^{3 *} \cup W_{i+1}^{2 *}\right)$. By symmetry, applying at most one bipartite complementation, we can remove all edges between vertices in $W_{i+1}^{2 *}$ and vertices in $V\left(H_{i}^{2}\right) \backslash\left(W_{i-1}^{3 *} \cup W_{i+1}^{2 *}\right)$. This disconnects $G\left[W_{i-1}^{3 *} \cup W_{i+1}^{2 *}\right]$ from the rest of $H_{i}^{2}$. Since $G\left[W_{i-1}^{3 *} \cup W_{i+1}^{2 *}\right]$ is a bipartite graph, it is curious. We may therefore assume that $W_{i-1}^{3}$ is anti-complete to $W_{i+1}^{2}$.

We set $X^{\prime}=\left(V_{i+3}^{0} \cup V_{i+3}^{+}\right) \cap V\left(H_{i}^{2}\right), Y^{\prime}=\left(V_{i+2}^{0} \cup V_{i+2}^{-}\right) \cap V\left(H_{i}^{2}\right)$ and $Z^{\prime}=\left(W_{i}^{0} \cup W_{i-1}^{3} \cup W_{i+1}^{2}\right) \cap V\left(H_{i}^{2}\right)$ and note that $X^{\prime}, Y^{\prime}$ and $Z^{\prime}$ are independent sets. Now suppose, for contradiction, that $H_{i}^{2}$ is not curious with respect to the partition $\left(X^{\prime}, Y^{\prime}, Z^{\prime}\right)$. Then there must be vertices $x \in X^{\prime}, y \in Y^{\prime}$ and $z \in Z^{\prime}$ such that $G[x, y, z]$ is a $3 P_{1}$. Since $x$ and $y$ are non-adjacent, it follows that $x \in V_{i+3}^{+}$and $y \in V_{i+2}^{-}$. By Claim 28, $z \notin W_{i}^{0}$. By symmetry, we may assume $z \in W_{i-1}^{3}$. Let $w$ be an arbitrary vertex of $W_{i}^{0}$. Then $w$ must be non-adjacent to $y$, otherwise $G\left[v_{i-1}, z, w, y, v_{i+1}, v_{i+2}\right]$ is a $P_{2}+P_{4}$. By Claim 28, $w$ must therefore be adjacent to $x$. Thus $G\left[v_{i+1}, y, w, x, v_{i-1}, z\right]$ is a $P_{2}+P_{4}$, a contradiction. It follows that $H_{i}^{2}$ is indeed a curious graph with respect to the partition $\left(X^{\prime}, Y^{\prime}, Z^{\prime}\right)$. This completes the proof of Claim 33.

Applying Claims 30, 31, 32 and 33, we remove all vertices of $V_{i}$ and $W_{i}$ except maybe for some sets $V_{i}^{0}$. By definition, each set $V_{i}^{0}$ is complete to $V_{i-1}^{0} \cup V_{i+1}^{0}$ and by Claim 2 it is anti-complete to $V_{i-2}^{0} \cup V_{i+2}^{0}$. We delete the five vertices of the cycle $C$ and then apply a bipartite complementation between any pair of non-empty sets $V_{i}^{0}$ and $V_{i+1}^{0}$. This will yield an independent set, which is a curious $\left(K_{3}, P_{2}+P_{4}\right)$-free graph where two parts of the partition are empty. It remains only to count the number of operations applied and the number of obtained curious and 3-uniform graphs.

As explained in the proof of Claim 25, by Claim 9, we apply at most five bipartite complementations to separate $G\left[U \cup V^{*}\right]$ from the rest of the graph. We then delete at most five vertices from $G\left[U \cup V^{*}\right]$ to obtain a graph that is either curious or 3-uniform. Next, as also explained in the proof of Claim 25, we can separate $G\left[W^{*}\right]$ from the rest of $G \backslash\left(V^{*} \cup U\right)$ by applying $2 \times 2=4$ bipartite complementations and obtain at most two curious graphs. Next, in Claim 27, for each $i \in\{1, \ldots, 5\}$, we delete at most 512 vertices. Deleting the five vertices of the cycle $C$ yields five more vertex deletions. Recall that for $i \in\{1, \ldots, 5\}$, we partitioned $W_{i}$ into $W_{i}^{0}, W_{i}^{2}$ and $W_{i}^{3}$ and partitioned $V_{i}$ into $V_{i}^{0}, V_{i}^{-}$and $V_{i}^{+}$, yielding 30 subsets of vertices altogether. In Claims 30 and 31, we apply bipartite complementations between certain pairs of these subsets to separate the graphs of both kinds. Thus, in these claims we apply at most $\left(\begin{array}{c}30 \\ 2\end{array}\right)=435$ bipartite complementations. For each $i \in\{1, \ldots, 5\}$, Claim 32 says that $H_{i}^{1}$ is a curious graph. In Claim 33, for each $i \in\{1, \ldots, 5\}$ we apply at most two bipartite complementations and obtain at most two curious graphs. Finally, we apply at most five bipartite complementations between non-empty sets $V_{i}^{0}$ and $V_{i+1}^{0}$, yielding at most one curious graph. This gives a total of $5+4+435+(5 \times 2)+5=459$ bipartite complementations, $5+(5 \times 512)+5=2570$ vertex deletions yielding at most $1+2+(5 \times 1)+(5 \times 2)+1=19$ curious graphs and at most one 3 -uniform graph. This completes the proof.

We can now prove our main result. Recall that it was already known [12] that the class of $\left(K_{3}, P_{1}+P_{5}\right)$-free graphs has bounded clique-width, but that it was not known that this class is well-quasi-ordered.

Theorem 5. For $H \in\left\{P_{2}+P_{4}, P_{1}+P_{5}\right\}$ the class of $\left(K_{3}, H\right)$-free graphs is well-quasi-ordered by the labelled induced subgraph relation and has bounded clique-width.

Proof. Let $H \in\left\{P_{2}+P_{4}, P_{1}+P_{5}\right\}$. By Lemmas 2 and 5, we need only consider prime graphs in the class of $\left(K_{3}, H\right)$-free graphs.

First, consider the bipartite (not necessarily prime) $\left(K_{3}, H\right)$-free graphs. The class of bipartite $\left(K_{3}, H\right)$-free graphs forms a subclass of the class of $\left(P_{7}, S_{1,2,3}\right)$-free bipartite graphs, since $H \subseteq_{i} P_{7}, S_{1,2,3}$. The class of $\left(P_{7}, S_{1,2,3}\right)$-free bipartite graphs is well-quasi-ordered by the labelled induced subgraph relation by Lemma 6 and has bounded clique-width by Theorem 3 . Therefore the class of bipartite $\left(K_{3}, H\right)$-free graphs is well-quasi-ordered by the labelled induced subgraph relation and has bounded clique-width. 
Next, consider the $C_{5}$-free non-bipartite prime $\left(K_{3}, H\right)$-free graphs. Since these graphs are not bipartite, each of them must contain an induced odd cycle $C_{k}$ for some odd $k \geq 3$. Since $\left(K_{3}, C_{5}, H\right)$-free graphs are $\left(C_{3}, C_{5}\right)$-free, it follows that $k \notin\{3,5\}$, and so $k \geq 7$. Since $\left(K_{3}, C_{5}, H\right)$-free graphs are $H$-free, it follows that they contain no induced cycles on eight or more vertices, and so $k=7$. Recall that a prime graph on at least three vertices cannot contain two vertices that are false twins, otherwise these two vertices would form a non-trivial module. Therefore, by Lemma 1 , and since $H \subseteq_{i} S_{1,2,3}$, the class of prime $\left(K_{3}, C_{5}, H\right)$-free graphs containing an induced $C_{7}$ consists of precisely the graph $C_{7}$. It follows that the class of $C_{5}$-free non-bipartite prime $\left(K_{3}, H\right)$-free graphs is well-quasi-ordered by the labelled induced subgraph relation and has bounded clique-width.

It remains to consider the prime $\left(K_{3}, H\right)$-free graphs that contain an induced $C_{5}$; note that such graphs are non-bipartite. By Lemmas 15 or 16 , given any prime $\left(K_{3}, H\right)$-free that contains an induced $C_{5}$, it is sufficient to apply at most a constant number of vertex deletions and bipartite complementation operations to obtain a graph that is a disjoint union of $\left(K_{3}, H\right)$-free curious graphs and (in the $H=P_{2}+P_{4}$ case) 3-uniform graphs. Since vertex deletions and bipartite complementations preserve boundedness of clique-width by Facts 1 and 3 and preserve well-quasi-orderability by the labelled induced subgraph relation by Lemma 4 , it is sufficient to show that $\left(K_{3}, H\right)$-free curious graphs and 3-uniform graphs have bounded clique-width and are well-quasi-ordered by the labelled induced subgraph relation. As observed above, the class of bipartite $\left(K_{3}, H\right)$-free graphs is well-quasi-ordered by the labelled induced subgraph relation and has bounded clique-width. By Theorem 4, it follows that the class of curious $\left(K_{3}, H\right)$-free graphs is well-quasi-ordered by the labelled induced subgraph relation and has bounded clique-width. Lemmas 8 and 9, respectively, imply that 3-uniform graphs are well-quasi-ordered by the labelled induced subgraph relation and have bounded clique-width. This completes the proof.

\section{State-of-the-art summaries for bigenic graph classes}

The class of $\left(\overline{P_{1}+P_{4}}, P_{2}+P_{3}\right)$-free graphs is the only bigenic graph class left for which Conjecture 1 still needs to be verified; see [15] for discussion of why only this case remains, but note that this can also be deduced from Theorems 6 and 7 below.

Open Problem 1. Is Conjecture 1 true for the class of $\left(H_{1}, H_{2}\right)$-free graphs when $H_{1}=\overline{P_{1}+P_{4}}$ and $H_{2}=P_{2}+P_{3}$ ?

The class of $\left(\overline{P_{1}+P_{4}}, P_{2}+P_{3}\right)$-free graphs is (up to an equivalence relation ${ }^{2}$ ) one of the six remaining bigenic graph classes for which well-quasi-orderability is still open [15] and one of the five bigenic graph classes for which boundedness of clique-width is still open [4,6]. To make our paper self-contained we recall two theorems from [15] and [6], respectively, which sum up our current knowledge on bigenic graph classes (including the new results of this paper). We also include the lists of corresponding open cases below.

Here is the state-of-the-art summary for well-quasi-orderability for bigenic graph classes.

Theorem 6. Let $\mathcal{G}$ be a class of graphs defined by two forbidden induced subgraphs. Then:

1. $\mathcal{G}$ is well-quasi-ordered by the labelled induced subgraph relation if it is equivalent to a class of $\left(H_{1}, H_{2}\right)$-free graphs such that one of the following holds:

(i) $\mathrm{H}_{1}$ or $\mathrm{H}_{2} \subseteq_{i} \mathrm{P}_{4}$;

(ii) $H_{1}=s P_{1}$ and $H_{2}=K_{t}$ for some $s, t \geq 1$;

(iii) $H_{1} \subseteq_{i} P_{1}+P_{3}$ and $\overline{H_{2}} \subseteq_{i} P_{1}+P_{5}, P_{2}+P_{4}$ or $P_{6}$;

(iv) $\mathrm{H}_{1} \subseteq_{i} 2 P_{1}+P_{2}$ and $\overline{H_{2}} \subseteq_{i} P_{2}+P_{3}$ or $P_{5}$.

2. $\mathcal{G}$ is not well-quasi-ordered by the induced subgraph relation if it is equivalent to a class of $\left(H_{1}, H_{2}\right)$-free graphs such that one of the following holds:

(i) neither $\mathrm{H}_{1}$ nor $\mathrm{H}_{2}$ is a linear forest;

(ii) $H_{1} \supseteq_{i} 3 P_{1}$ and $\overline{H_{2}} \supseteq_{i} 3 P_{1}+P_{2}, 3 P_{2}$ or $2 P_{3}$;

(iii) $H_{1} \supseteq_{i} 2 P_{2}$ and $\overline{H_{2}} \supseteq_{i} 4 P_{1}$ or $2 P_{2}$;

(iv) $\mathrm{H}_{1} \supseteq_{i} 2 P_{1}+P_{2}$ and $\overline{H_{2}} \supseteq_{i} 4 P_{1}, P_{2}+P_{4}$ or $P_{6}$;

(v) $H_{1} \supseteq_{i} P_{1}+P_{4}$ and $\overline{H_{2}} \supseteq_{i} P_{1}+2 P_{2}$.

Theorem 6 shows that our result on the well-quasi-orderability of the class of $\left(K_{3}, P_{2}+P_{4}\right)$-free graphs is tight in the following sense: for every $H \neq \overline{P_{1}+P_{3}}$ that contains $K_{3}$, the class of $\left(H, P_{2}+P_{4}\right)$-free graphs is not well-quasi-ordered by the induced subgraph relation. In particular, this follows from [33] if $H \in\left\{K_{4}, K_{3}+P_{1}\right\}$ and from [15] if $H=\overline{2 P_{1}+P_{2}}$.

\footnotetext{
2 Given four graphs $\mathrm{H}_{1}, \mathrm{H}_{2}, \mathrm{H}_{3}, \mathrm{H}_{4}$, the classes of $\left(\mathrm{H}_{1}, \mathrm{H}_{2}\right)$-free graphs and $\left(\mathrm{H}_{3}, \mathrm{H}_{4}\right)$-free graphs are said to be equivalent if the unordered pair $\mathrm{H}_{3}, \mathrm{H}_{4}$ can be obtained from the unordered pair $\mathrm{H}_{1}, \mathrm{H}_{2}$ by some combination of the operations (i) complementing both graphs in the pair and (ii) if one of the graphs in the pair is $3 P_{1}$, replacing it with $P_{1}+P_{3}$ or vice versa. If two classes are equivalent, then one of them is well-quasi-ordered by the induced subgraph relation if and only if the other one is [33]. Similarly, if two classes are equivalent, then one of them has bounded clique-width if and only if the other one does [17].
} 
Theorem 6 does not cover six cases, which are all still open.

Open Problem 2. Is the class of $\left(H_{1}, H_{2}\right)$-free graphs well-quasi-ordered by the induced subgraph relation when:

(i) $H_{1}=2 P_{1}+P_{2}$ and $\overline{H_{2}} \in\left\{P_{1}+2 P_{2}, P_{1}+P_{4}\right\}$;

(ii) $H_{1}=P_{1}+P_{4}$ and $\overline{H_{2}} \in\left\{P_{1}+P_{4}, 2 P_{2}, P_{2}+P_{3}, P_{5}\right\}$ ?

Here is the state-of-the-art summary of the boundedness of clique-width for bigenic graph classes.

Theorem 7. Let $\mathcal{G}$ be a class of graphs defined by two forbidden induced subgraphs. Then:

1. $\mathcal{G}$ has bounded clique-width if it is equivalent to a class of $\left(\mathrm{H}_{1}, \mathrm{H}_{2}\right)$-free graphs such that one of the following holds:

(i) $\mathrm{H}_{1}$ or $\mathrm{H}_{2} \subseteq_{i} \mathrm{P}_{4}$

(ii) $H_{1}=K_{s}$ and $H_{2}=t P_{1}$ for some $s, t \geq 1$

(iii) $H_{1} \subseteq_{i} \overline{P_{1}+P_{3}}$ and $H_{2} \subseteq_{i} K_{1,3}+3 P_{1}, K_{1,3}+P_{2}, P_{1}+P_{2}+P_{3}, P_{1}+P_{5}, P_{1}+S_{1,1,2}, P_{2}+P_{4}, P_{6}, S_{1,1,3}$ or $S_{1,2,2}$

(iv) $H_{1} \subseteq_{i} \overline{2 P_{1}+P_{2}}$ and $H_{2} \subseteq_{i} P_{1}+2 P_{2}, 3 P_{1}+P_{2}$ or $P_{2}+P_{3}$

(v) $H_{1} \subseteq_{i} \overline{P_{1}+P_{4}}$ and $H_{2} \subseteq_{i} P_{1}+P_{4}$ or $P_{5}$

(vi) $H_{1} \subseteq_{i} K_{3}+P_{1}$ and $H_{2} \subseteq_{i} K_{1,3}$, or

(vii) $H_{1} \subseteq_{i} \overline{2 P_{1}+P_{3}}$ and $H_{2} \subseteq_{i} 2 P_{1}+P_{3}$.

2. $\mathcal{G}$ has unbounded clique-width if it is equivalent to a class of $\left(H_{1}, H_{2}\right)$-free graphs such that one of the following holds:

(i) $H_{1} \notin \mathcal{S}$ and $H_{2} \notin \mathcal{S}$

(ii) $H_{1} \notin \overline{\mathcal{S}}$ and $\mathrm{H}_{2} \notin \overline{\mathcal{S}}$

(iii) $H_{1} \supseteq_{i} K_{3}+P_{1}$ or $C_{4}$ and $H_{2} \supseteq_{i} 4 P_{1}$ or $2 P_{2}$

(iv) $H_{1} \supseteq_{i} \overline{2 P_{1}+P_{2}}$ and $H_{2} \supseteq_{i} K_{1,3}, 5 P_{1}, P_{2}+P_{4}$ or $P_{6}$

(v) $H_{1} \supseteq_{i} K_{3}$ and $H_{2} \supseteq_{i} 2 P_{1}+2 P_{2}, 2 P_{1}+P_{4}, 4 P_{1}+P_{2}, 3 P_{2}$ or $2 P_{3}$

(vi) $H_{1} \supseteq_{i} K_{4}$ and $H_{2} \supseteq_{i} P_{1}+P_{4}$ or $3 P_{1}+P_{2}$, or

(vii) $H_{1} \supseteq_{i} \overline{P_{1}+P_{4}}$ and $H_{2} \supseteq_{i} P_{1}+2 P_{2}$.

Theorem 7 shows that our result on the boundedness of clique-width of the class of $\left(K_{3}, P_{2}+P_{4}\right)$-free graphs is tight in the following sense: for every $H \neq \overline{P_{1}+P_{3}}$ that contains $K_{3}$, the class of $\left(H, P_{2}+P_{4}\right)$-free graphs has unbounded clique-width. In particular, this follows from [7] if $H \in\left\{K_{4}, K_{3}+P_{1}\right\}$ and from [13] if $H=\overline{2 P_{1}+P_{2}}$.

Theorem 7 does not cover five cases, which are all still open.

Open Problem 3. Does the class of $\left(H_{1}, H_{2}\right)$-free graphs have bounded or unbounded clique-width when:

(i) $H_{1}=K_{3}$ and $H_{2} \in\left\{P_{1}+S_{1,1,3}, S_{1,2,3}\right\}$

(ii) $H_{1}=\overline{2 P_{1}+P_{2}}$ and $H_{2} \in\left\{P_{1}+P_{2}+P_{3}, P_{1}+P_{5}\right\}$

(iii) $H_{1}=\overline{P_{1}+P_{4}}$ and $H_{2}=P_{2}+P_{3}$ ?

\section{Conclusions}

In this paper, we made a contribution to answering the recently posed question on the relationship between two seemingly unrelated notions: well-quasi-orderability by the induced subgraph relation and boundedness of clique-width, for hereditary graph classes. In particular, we showed that in the case of two forbidden induced subgraphs, one of which is the triangle, well-quasi-orderability by the induced subgraph relation implies boundedness of clique-width. In order to do this, we exploited similarities in the techniques for showing well-quasi-orderability by the induced subgraph relation and boundedness of clique-width.

In general, the relationship between the two notions remains largely unexplored and Conjecture 1 is still open. A major part of the difficulty of either proving Conjecture 1 or constructing a counter-example to it is a lack of understanding of the structure of graph classes that are not well-quasi-ordered by the induced subgraph relation. Investigating the structure of infinite antichains may lead to new tools for detecting infinite antichains. This could help us with deducing new and more decisive properties for a graph class to be well-quasi-ordered and/or to have bounded clique-width. We leave this agenda for future research.

\section{Declaration of competing interest}

The authors declare that they have no known competing financial interests or personal relationships that could have appeared to influence the work reported in this paper. 


\section{Acknowledgments}

\section{We thank two anonymous reviewers for helpful comments.}

\section{References}

[1] P.A. Abdulla, K. Čerāns, B. Jonsson, Y.-K. Tsay, Algorithmic analysis of programs with well quasi-ordered domains, Inf. Comput. 160 (1-2) (2000) $109-127$.

[2] P. Allen, V.V. Lozin, M. Rao, Clique-width and the speed of hereditary properties, Electron. J. Comb. 16 (1) (2009), Research Paper 35, 11 pp.

[3] A. Atminas, V.V. Lozin, Labelled induced subgraphs and well-quasi-ordering, Order 32 (3) (2015) 313-328.

[4] A. Blanché, K.K. Dabrowski, M. Johnson, V.V. Lozin, D. Paulusma, V. Zamaraev, Clique-width for graph classes closed under complementation, in: Proc. MFCS 2017, in: LIPIcs, vol. 83, 2017, 73, 14 pp.

[5] J. Błasiok, M. Kamiński, J.-F. Raymond, T. Trunck, Induced minors and well-quasi-ordering, J. Comb. Theory, Ser. B 134 (2019) 110-142.

[6] M. Bonamy, K.K. Dabrowski, M. Johnson, D. Paulusma, Graph isomorphism for $\left(H_{1}, H_{2}\right)$-free graphs: an almost complete dichotomy, in: Proc. WADS 2019, in: LNCS, vol. 11646, 2019, pp. 181-195.

[7] A. Brandstädt, J. Engelfriet, H.-O. Le, V.V. Lozin, Clique-width for 4-vertex forbidden subgraphs, Theory Comput. Syst. 39 (4) (2006) 561-590.

[8] R. Brignall, M. Engen, V. Vatter, A counterexample regarding labelled well-quasi-ordering, Graphs Comb. 34 (6) (2018) $1395-1409$.

[9] B. Courcelle, Clique-width and edge contraction, Inf. Process. Lett. 114 (1-2) (2014) 42-44.

[10] B. Courcelle, J.A. Makowsky, U. Rotics, Linear time solvable optimization problems on graphs of bounded clique-width, Theory Comput. Syst. 33 (2) (2000) 125-150.

[11] B. Courcelle, S. Olariu, Upper bounds to the clique width of graphs, Discrete Appl. Math. 101 (1-3) (2000) 77-114.

[12] K.K. Dabrowski, F. Dross, D. Paulusma, Colouring diamond-free graphs, J. Comput. Syst. Sci. 89 (2017) $410-431$.

[13] K.K. Dabrowski, S. Huang, D. Paulusma, Bounding clique-width via perfect graphs, J. Comput. Syst. Sci. 104 (2019) $202-215$.

[14] K.K. Dabrowski, V.V. Lozin, D. Paulusma, Clique-width and well-quasi ordering of triangle-free graph classes, in: Proc. WG 2017, in: LNCS, vol. 10520, 2017, pp. 220-233.

[15] K.K. Dabrowski, V.V. Lozin, D. Paulusma, Well-quasi-ordering versus clique-width: new results on bigenic classes, Order 35 (2) (2018) $253-274$.

[16] K.K. Dabrowski, D. Paulusma, Classifying the clique-width of $H$-free bipartite graphs, Discrete Appl. Math. 200 (2016) $43-51$.

[17] K.K. Dabrowski, D. Paulusma, Clique-width of graph classes defined by two forbidden induced subgraphs, Comput. J. 59 (5) (2016) 650-666.

[18] J. Daligault, M. Rao, S. Thomassé, Well-quasi-order of relabel functions, Order 27 (3) (2010) $301-315$.

[19] P. Damaschke, Induced subgraphs and well-quasi-ordering, J. Graph Theory 14 (4) (1990) 427-435.

[20] G. Ding, Subgraphs and well-quasi-ordering, J. Graph Theory 16 (5) (1992) 489-502.

[21] W. Espelage, F. Gurski, E. Wanke, How to solve NP-hard graph problems on clique-width bounded graphs in polynomial time, in: Proc. WG 2001, in: LNCS, vol. 2204, 2001, pp. 117-128.

[22] M.R. Fellows, D. Hermelin, F.A. Rosamond, Well quasi orders in subclasses of bounded treewidth graphs and their algorithmic applications, Algorithmica 64 (1) (2012) 3-18

[23] A. Finkel, P. Schnoebelen, Well-structured transition systems everywhere!, Theor. Comput. Sci. 256 (1-2) (2001) 63-92

[24] J.-L. Fouquet, V. Giakoumakis, J.-M. Vanherpe, Bipartite graphs totally decomposable by canonical decomposition, Int. J. Found. Comput. Sci. 10 (04) (1999) 513-533.

[25] P.A. Golovach, M. Johnson, D. Paulusma, J. Song, A survey on the computational complexity of colouring graphs with forbidden subgraphs, J. Graph Theory 84 (4) (2017) 331-363.

[26] F. Gurski, The behavior of clique-width under graph operations and graph transformations, Theory Comput. Syst. 60 (2) (2017) 346-376.

[27] G. Higman, Ordering by divisibility in abstract algebras, Proc. Lond. Math. Soc. s3-2 (1) (1952) 326-336.

[28] K. ichi Kawarabayashi, Y. Kobayashi, B. Reed, The disjoint paths problem in quadratic time, J. Comb. Theory, Ser. B 102 (2) (2012) $424-435$.

[29] M. Kamiński, V.V. Lozin, M. Milanič, Recent developments on graphs of bounded clique-width, Discrete Appl. Math. 157 (12) (2009) $2747-2761$.

[30] M. Kamński, J.-F. Raymond, T. Trunck, Well-quasi-ordering H-contraction-free graphs, Discrete Appl. Math. 248 (2018) $18-27$.

[31] D. Kobler, U. Rotics, Edge dominating set and colorings on graphs with fixed clique-width, Discrete Appl. Math. 126 (2-3) (2003) 197-221.

[32] N. Korpelainen, V.V. Lozin, Bipartite induced subgraphs and well-quasi-ordering, J. Graph Theory 67 (3) (2011) $235-249$.

[33] N. Korpelainen, V.V. Lozin, Two forbidden induced subgraphs and well-quasi-ordering, Discrete Math. 311 (16) (2011) $1813-1822$.

[34] N. Korpelainen, V.V. Lozin, I. Razgon, Boundary properties of well-quasi-ordered sets of graphs, Order 30 (3) (2013) $723-735$.

[35] J.B. Kruskal, The theory of well-quasi-ordering: a frequently discovered concept, J. Comb. Theory, Ser. A 13 (3) (1972) $297-305$

[36] V.V. Lozin, D. Rautenbach, On the band-, tree-, and clique-width of graphs with bounded vertex degree, SIAM J. Discrete Math. 18 (1) (2004) $195-206$.

[37] V.V. Lozin, I. Razgon, V. Zamaraev, Well-quasi-ordering versus clique-width, J. Comb. Theory, Ser. B 130 (2018) 1-18.

[38] W. Mader, Wohlquasigeordnete Klassen endlicher Graphen, J. Comb. Theory, Ser. B 12 (2) (1972) $105-122$.

[39] S. Oum, P.D. Seymour, Approximating clique-width and branch-width, J. Comb. Theory, Ser. B 96 (4) (2006) 514-528.

[40] F.P. Ramsey, On a problem of formal logic, Proc. Lond. Math. Soc. s2-30 (1) (1930) 264-286.

[41] M. Rao, MSOL partitioning problems on graphs of bounded treewidth and clique-width, Theor. Comput. Sci. 377 (1-3) (2007) 260-267.

[42] N. Robertson, P.D. Seymour, Graph minors. XIII. The disjoint paths problem, J. Comb. Theory, Ser. B 63 (1) (1995) 65-110.

[43] N. Robertson, P.D. Seymour, Graph minors. XX. Wagner’s conjecture, J. Comb. Theory, Ser. B 92 (2) (2004) $325-357$. 